\title{
REGULATION AND CAPACITY COMPETITION IN HEALTH CARE: EVIDENCE FROM U.S. DIALYSIS MARKETS
}

\author{
Mian Dai and Xun Tang*
}

\begin{abstract}
This paper studies entry and capacity decisions by dialysis providers in the United States. We estimate a structural model where providers make continuous strategic choices of capacity based on their private information about own costs and knowledge of the distribution of competitors' private information. We evaluate the impact on the market structure and providers' profits under counterfactual regulatory policies that increase the costs or reduce the payment per unit of capacity. We find that these policies reduce the market capacity as measured by the number of dialysis stations. However, the downward-sloping reaction curve shields some providers from negative profit shocks in certain markets. The paper also has a methodological contribution in that it proposes new estimators for Bayesian games with continuous actions.
\end{abstract}

\section{Introduction}

$\mathrm{D}$ IALYSIS is the major treatment for more than 630,000 patients in the United States with end-stage renal disease (ESRD). Medicare, the monopolistic buyer of dialysis services, spent $\$ 8.6$ billion in 2007 on the treatment and medication of dialysis patients. While Medicare pays a fixed rate to dialysis providers, rising dialysis expenditure motivates a recent Medicare reform that aims at reducing costs and maintaining the quality of care. ${ }^{1}$ The core component of the reform is a new reimbursement system that incorporates payments for multiple drugs and services furnished in a dialysis session into a single bundled rate. This effectively reduces the average per treatment rate received by dialysis providers and subsequently lowers the per patient margin. Lowering the dialysis reimbursement rate also raises the concern of insufficient payment, which may eventually compromise dialysis accessibility. Dialysis capacity, as measured by the number of dialysis stations, is an important metric that policymakers use to evaluate the adequacy of dialysis payment policies. In this paper, we analyze the provision of dialysis capacity and evaluate the implications of counterfactual dialysis payment policies. Our results offer some insights into the conduct of health care providers and the effectiveness of fixed-price regulation.

We build a model of static Bayesian games with continuous actions to examine the strategic interactions between U.S. dialysis providers in their choices of capacity across Hospital Service Areas (HSA) in 2007. We focus on three types of providers on the market: FMC, DaVita, and all other

Received for publication July 15, 2013. Revision accepted for publication February 13, 2014. Editor: Philippe Aghion.

* Dai: Drexel University; Tang: Rice University.

We are grateful to the editor and two anonymous referees for thoughtful and constructive comments. We also thank Ulrich Doraszelski, Hanming Fang, Jean-François Houde, Katja Seim, Mark Stehr, and Robert Town for helpful comments and suggestions and Ryan McDevitt for kind assistance with data. All remaining errors are our own.

${ }^{1}$ The reform was proposed in 2008 and became effective in 2011. The full implementation of the policy was completed in 2014. nonchain providers. ${ }^{2}$ We estimate how payoffs of these providers depend on exogenous market characteristics (such as size and measures of ESRD risks) as well as the endogenous choices of capacity. We then use our estimates to predict the impact of payment policies that either raise or reduce the margin per unit of capacity. In particular, we are interested in how different providers would respond to positive or negative adjustment in the reimbursement for dialysis treatment and whether high capacity helps dialysis providers maintain their market presence with lower margins.

The impact of strategic choices of capacity on the market structure and the intensity of competition has been studied in several theoretical papers (Dixit, 1980; Gelman \& Salop, 1983; Kreps \& Scheinkman 1983). The empirical literature, however, has largely ignored the strategic incentives in the continuous choices of capacity. With little price and quality competition (as shown in Grieco \& McDevitt, 2013, and Cutler et al., 2012), these strategic incentives are important for dialysis providers. ${ }^{3}$ In practice, a dialysis provider's margin decreases in the capacity of competitors. One explanation for this pattern is that additional capacity allows a provider to offer a more flexible treatment schedule, which patients highly value. ${ }^{4}$ Given the high operation and maintenance costs for each dialysis station, optimal capacity choices depend on the trade-off among market demand, capacity costs, and competitive interactions between dialysis providers.

We characterize providers' strategies in a Bayesian Nash equilibrium by the first-order conditions of their constrained maximization problems (subject to the constraints of nonnegative capacity). We show that these conditions are similar to censored regressions, except that the expected capacity for competitors in equilibrium now enters as a "generated" regressor. We propose several estimators (two-stage, MLE, and GMM) to infer the marginal effects of a provider's own capacity, the capacity of its competitors, and the market conditions on its profits.

Our estimates conform to the empirical regularities that a dialysis provider's choice of capacity is decreasing in its

\footnotetext{
${ }^{2}$ Fresenius Medical Care (FEC) and DaVita are the industry leaders with national footprints. They jointly own two-thirds of the dialysis facilities and treat more than two-thirds of all dialysis patients. We refer to other non-FMC or non-DaVita providers as nonchain providers. The scale of operation and the market penetration of each of these nonchain providers are not comparable to the two leaders.

${ }^{3}$ Both Grieco and McDevitt (2014) and Cutler, Dafny, and Ody (2012) find little effect of competition on various measures of dialysis quality and patient outcomes.

${ }^{4}$ Given that a typical dialysis patient visits providers three times a week for a total of nine to twelve hours, the scheduling flexibility is found to be more important than the survival rate in patient's dialysis choice (Johansen, 2011). It is easier for patients to find their preferred appointments with the providers capable of performing multiple concurrent dialysis sessions.
} 
competitors' choices of capacity (i.e., the reaction curve is downward sloping) and that competition is more intense between competitors with high capacity. We find that all else being equal, increasing a provider's expected capacity by 1 unit (a dialysis station) reduces a competitor's choice of capacity by an average of 0.2 to 0.4 units and decreases the competitor's entry probability by $3 \%$ to $13 \%$ in equilibrium. This suggests that focusing on providers' binary entry decisions overlooks rather substantial strategic interactions between their choices of capacity. Our results are also robust to various sample selection criteria and different methods of econometric implementation (such as two-stage, nested maximum-likelihood, and GMM).

In our counterfactual analyses, we find that on average, providers' capacity choices respond negatively to a reduction in the profit margin that results from a more stringent reimbursement policy. The effects are reversed under more generous reimbursement policies that increase the profit margin. More interesting, the responses are heterogeneous across markets and providers. For example, FMC and nonchain independent providers respond most strongly to both negative and positive margin adjustments, while DaVita reacts mildly in both cases. In some markets, DaVita reduces (increases) its capacity when the margin widens (shrinks) by the same portion as its competitors. Such a pattern is explained by the downward-sloping reaction curve in the Bayesian game of capacity choices, which incentivizes DaVita to decrease its capacity if the competitors expand and increase its capacity if others scale down. Our results show that the effects of the reaction curve are large enough to offset the impact of positive (negative) adjustments in the profit margins in certain markets. This offers evidence that the shape of the reaction curve could potentially magnify a provider's response to small changes in the profit margin in some markets and that it plays a crucial role in determining the effect of reimbursement policies.

Apart from its empirical motivation and findings, our paper also contributes to the methodological literature on the estimation of empirical games. We are not aware of any previous work structurally estimating Bayesian games with continuous actions. The bulk of existing literature studies competitive effects in games with discrete actions in various contexts such as entry games, where alternatives available to players are naturally finite (Aradillas-Lopez, 2010; Bajari et al., 2010; Berry \& Tamer, 2006; Davis, 2006; Mazzeo, 2002; Seim, 2006; Sweeting, 2009). In our application, binary entry decisions are closely associated with continuous choices of capacity, suggesting that even small incremental changes in capacity play an important role in determining the firms' payoffs and the market outcomes. One of the main messages of our paper is that overlooking the information revealed in the chosen level of capacity may compromise researchers' understanding of the market mechanism.

The rest of this paper is organized as follows. Section II introduces the background of the dialysis industry. Section
III specifies the econometric model for our structural analysis. Section IV discusses the identification and estimation of the model. Section V describes the data. Section VI presents empirical findings and results from the counterfactual exercises. Section VII concludes.

\section{Background}

\section{A. Dialysis and Capacity}

Chronic kidney disease (CKD) affects more than 20 million adults in the United States. The advanced stage of CKD, known as end-stage renal disease (ESRD), is most commonly caused by diabetes and high blood pressure. The only treatment for it is dialysis or a kidney transplant. Given the limited supply of donor organs and the surgical risk associated with comorbidity, many ESRD patients rely on routine dialysis as the major treatment.

Dialysis removes waste (such as urea) and excess water from the body as a replacement for lost kidney function. Hemodialysis is the most common treatment modality and accounts for about $90 \%$ of the dialysis population in the United States. ${ }^{5}$ In a hemodialysis session, a dialysis machine pumps a patient's blood into the dialyzer, cleans it with dialysate (a solution that removes excess fluids and wastes), and injects the cleaned blood back into the patient's body. It is impossible to perform dialysis without those machines.

Acquiring and operating a dialysis machine is costly. A new dialysis machine costs between $\$ 10,000$ and $\$ 15,000$ and has a life span of five to seven years. Among the associated costs are dialysis chairs and privacy screens. Industry experts estimate a cost of $\$ 100,000$ to maintain and operate one dialysis station over its lifetime. The dialysis capacity in a given market (HSA), as measured by the number of dialysis stations operated, is practically a permanent decision for each dialysis provider; the data available typically report little subsequent adjustment in capacity by providers following initial entry into a market. Grieco and McDevitt (2014) find that dialysis capacity remained constant for over $90 \%$ of the dialysis facilities in the United States between 2004 and 2007.

\section{B. Regulatory Background}

ESRD patients receive almost universal coverage under Medicare regardless of their age. ${ }^{6}$ Around $80 \%$ of the dialysis population relies on Medicare as the primary payer (U.S. Renal Data System, 2010). Under the old system, Medicare reimbursed three dialysis sessions per week under a fixed rate, after adjusting for the patient's case mix, local wages, and other factors associated with the cost of treatment. In addition, providers are paid for separately billable services that are furnished during the in-center hemodialysis sessions (e.g., injectable drugs such as Epogen and diagnostic

\footnotetext{
5 Alternatively, about $10 \%$ of the dialysis population chooses peritoneal dialysis, which patients usually perform every day at home.

6 ESRD was recognized as disability under the Medicare Reform Act in 1972. The legislation was signed into law and became effective in 1973.
} 
laboratory tests), which represent about $40 \%$ of total Medicare payments per dialysis treatment session. The generous reimbursement for separately billable services has raised the concern that it may create distorted profit incentives for the overuse of drugs. For example, one of the separately billable drugs, Epogen, primarily used for treating a common complication of ESRD, cost \$2.1 billion in 2008 and has become Medicare's largest drug expenditure. DaVita, one of the largest chain dialysis providers, was investigated for overbilling dialysis drugs. ${ }^{7}$ Thamer et al. (2007) found that large profit-seeking chain facilities used larger doses of Epogen and suggested this was due to the profit incentives. The excessive use of such drugs not only increased Medicare costs but also raised cardiovascular risk (e.g., heart attack, stroke) and subsequently lowered the life quality of ESRD patients.

In 2008, Medicare proposed a new payment system, eliminating the drug incentive by incorporating separate billable items into an expanded bundled payment. ${ }^{8}$ Additionally, payfor-performance quality incentives were introduced under the new system. Dialysis providers whose quality of service (as measured by patients' hemoglobin and urea levels) fails to meet standards could be penalized with a rate reduction of up to $2 \%$. The new dialysis payment system became effective in 2011, and full implementation took place in 2014.

The new Medicare reimbursement rule could have a significant impact on dialysis providers. The Government Accountability Office estimates $\$ 880$ million in savings on dialysis payments. ${ }^{9}$ Our counterfactual experiments are motivated by this reform and investigate how dialysis providers respond to different adjustments in the reimbursement rate.

\section{Dialysis Market}

The dialysis market in the United States can be characterized as a duopoly market. In 2007, DaVita and Fresenius Medical Care (FMC), the two largest national chains, treated over $66 \%$ of dialysis patients $(31 \%$ by DaVita versus $35 \%$ by FMC) and owned around $66 \%$ of the dialysis facilities ( $30 \%$ by DaVita and $36 \%$ by FMC). ${ }^{10}$ Both chains grew significantly after a series of consolidations in the 2000s. In 2004, DaVita bought Gambro, which owned over 550 facilities, and in 2005, FMC bought Renal Care Group with more than 450 facilities. ${ }^{11}$ Overall, the mergers between 2004 and 2006 consolidated the six largest chains into two. As of 2007, the market has been dominated by these two major chain providers.

\footnotetext{
${ }^{7}$ In 2006, 25\% of DaVita's revenue came from Epogen. The government decided not to pursue the case in 2011.

${ }^{8}$ For example, the Medicare base rate per dialysis session was $\$ 133.81$, while it was $\$ 229.63$ under the 2008 proposal. Note that the proposed base rate incorporates all separable billable services, including lab test and injectable drugs.

$9 \mathrm{http} / / /$ www.ama-assn.org/amednews/2012/12/24/gvsd1226.htm.

${ }^{10}$ U.S. Renal Data System (2009).

11 The merger between FMC and Renal Care Group was announced in May 2005 and completed in March 2006.
}

We focus on three types of dialysis providers: FMC, DaVita, and all other providers (referred to as nonchain providers). Given the proximity in the time frame between 2004 and 2006 when these major consolidations and subsequent market reorganization took place, we believe that the decisions of providers can be treated as made simultaneously. Using information on the capacity of dialysis providers between 2004 and 2009, we find that both DaVita and FMC adjusted their choices of capacity in the acquired facilities immediately following the merger and that capacity in the acquired facilities has barely changed since 2007 . These facts offer evidence that the providers' choices of capacity reported in data should be interpreted as equilibrium outcomes.

\section{A Model of Capacity Choices with Private Information}

We now specify a model of simultaneous Bayesian games with continuous actions. Consider a market that is served by $N$ providers competing through the choices of capacity. A provider (firm) $i$ 's profit from choosing capacity $K_{i}$ in a market $m$, as measured by the number of dialysis stations, is given by

$$
\begin{aligned}
& \Pi_{i, m}\left(K_{i, m}, K_{-i, m}, X_{m}, \varepsilon_{i, m}\right) \\
& \quad=K_{i, m} \pi_{i}\left(X_{m}, K_{-i, m}, \varepsilon_{i, m}\right)-c_{i}\left(K_{i, m}\right) .
\end{aligned}
$$

The function $\pi_{i}\left(X_{m}, K_{-i, m}, \varepsilon_{i, m}\right)$ is the variable profit for $i$ per unit in capacity (a provider's variable profit per unit in a market is defined as the ratio between its gross profits before subtracting the fixed costs in $c_{i}$ and its total capacity in that market.) It depends on the vector of competitors' capacity $K_{-i, m} \equiv\left(K_{j, m}\right)_{j \neq i}$, market characteristics $X_{m}$, and an idiosyncratic profit component $\varepsilon_{i, m}$, which is $i$ 's private information. We assume $\varepsilon_{i, m}$ are independent across providers conditional on $X_{m}$. To simplify notation, we drop the subscript $m$ below.

An implication of the specification is that the gross variable profit for a provider is proportional to its choice of capacity. This is motivated by the observation that each dialysis machine receives a flat rate from Medicare for each treatment. A typical dialysis patient receives three treatments per week, each lasting for about 4 hours. An additional hour is needed for setting up and cleaning the machine per treatment. This sums to 15 hours per week. On average, a dialysis machine treats three to five patients per week. During a treatment, operating staff such as registered nurses and technicians need to oversee the process and perform routine checks. Given the fixed rate for a dialysis treatment, it is plausible that a provider's variable profit is approximately proportional to its dialysis capacity, as measured by the number of dialysis stations.

We adopt a linear specification of per unit variable profit $\pi_{i}$,

$$
\pi_{i}\left(X, K_{-i}, \varepsilon_{i}\right)=X \beta_{i}+\sum_{j \neq i} \gamma_{i, j} K_{j}-\varepsilon_{i},
$$


where $\gamma_{i, j}$ are heterogeneous marginal effects of $j$ 's capacity on $i$ 's per unit variable profits. There are two reasons for this simplistic specification.

First, this linear specification for $\pi_{i}$ can be interpreted as a practical reduced-form approximation (regression) of actual variable profit per unit in capacity in the data-generating process. Using this as a benchmark helps us to understand how strategic capacity choices determine market outcome and firms' profits. As we show in section VI, this specification explains a large portion of the variation in capacity choices reported in the data. It is worth noting that a provider's own capacity is not included in the specification for per capacity variable profits $\pi_{i}$. This means the gross variable profits are proportional rather than quadratic in a provider's own capacity. Such proportionality is mostly motivated by the inelastic market demand for dialysis treatment and Medicare's fixed rates for dialysis treatment.

Second, focusing on this linear specification allows us to establish the identification of marginal effects of market characteristics on profits. In principle, we could extend our estimation algorithm in section IV to a richer structural model (such as one with market-level unobserved heterogeneity) in order to fit the data better. Yet this is known to raise new challenges with the identification of structural parametersin particular, the marginal effects of capacity and market conditions on providers' profits.

The firm-specific fixed cost is given by

$$
c_{i}\left(K_{i}\right)=a_{i} K_{i}^{2}+b_{i} K_{i}, \text { where } a_{i}>0
$$

We adopt a quadratic cost specification for the following reasons. First, the assumption of constant scale of economy (i.e., costs are linear in capacity) is not plausible in the dialysis industry. Adding a dialysis station not only involves significant investment but also requires additional space, maintenance, and personnel (e.g., technicians, nurses), whose supply is relatively inelastic. The quadratic form is helpful to capture such features as well. Second, quadratic costs take a flexible nonlinear form and thus can be considered a secondorder polynomial approximation to more complicated cost structures. Our estimation algorithm in section IV can be extended to allow higher-order polynomials in the specification. Finally, that there is no constant term in the quadratic function is due to the need for a location normalization: profits from no entry $\left(K_{i}=0\right)$ need to be 0 .

We assume that choices of capacity in the data are rationalized by providers' strategies in a pure strategy Bayesian Nash equilibrium (PBNE). A pure strategy for a provider $i$ is a mapping from its information set $\left(X, \varepsilon_{i}\right)$ into the support for capacity $\left(\mathbb{R}_{+}\right)$. A profile of pure strategies $\left\{K_{i}^{*}(.)\right\}_{i \in N}$ forms a PBNE if

$$
\begin{aligned}
& K_{i}^{*}\left(X, \varepsilon_{i}\right) \\
& \quad=\arg \max _{K_{i} \in \mathbb{R}_{+}} \mathbb{E}_{\varepsilon_{-i}}\left[\Pi_{i}\left(K_{i}, K_{-i}^{*}\left(X, \varepsilon_{-i}\right), X, \varepsilon_{i}\right) \mid X, \varepsilon_{i}\right],
\end{aligned}
$$

where $K_{-i}^{*}\left(X, \varepsilon_{-i}\right)$ is shorthand for $\left\{K_{j}^{*}\left(X, \varepsilon_{j}\right): j \neq i\right\}$. The case with $K_{i}=0$ means that provider $i$ decides not to enter the market. The existence of PBNE in our model follows from theorem 3 in Athey (2001) and the fact that the crossderivatives of the ex post profit for $i$ with respect to realized choices of capacity $\left(K_{i}, K_{j}\right)$ and $\left(K_{i}, \varepsilon_{j}\right)$ are constants.

Next, we derive the first-order condition for PBNE, the foundation for our estimators. We maintain a conventional regularity condition that ensures the order of differentiation and integration in $\frac{\partial}{\partial K_{i}} \mathbb{E}_{\varepsilon_{-i}}\left[\Pi_{i}\left(K_{i}, K_{-i}\left(X, \varepsilon_{-i}\right), X, \varepsilon_{i}\right) \mid X, \varepsilon_{i}\right]$ can be switched for all $i$ and vectors of admissible strategies $K_{-i}\left(X, \varepsilon_{-i}\right)$.

\section{Proposition 1. Under the model assumptions above,}

$$
\begin{aligned}
K_{i}^{*}\left(X, \varepsilon_{i}\right) & =\max \left\{0, \frac{1}{2 a_{i}}\right. \\
& \left.\left(X \beta_{i}+\sum_{j \neq i} \gamma_{i, j} \mathbb{E}_{\varepsilon_{j}}\left[K_{j}^{*}\left(X, \varepsilon_{j}\right) \mid X, \varepsilon_{i}\right]-b_{i}-\varepsilon_{i}\right)\right\}
\end{aligned}
$$

\section{in any $P B N E$.}

This proposition has a couple of key implications for estimation. First, it implies that the scale of $a_{i}, b_{i}, \beta_{i}, \gamma_{i, j}$ and the distribution of $\varepsilon_{i}$ cannot be jointly recovered from equation (3). Hence without loss of generality, we set $a_{i}=1 / 2$ as a necessary scale normalization. ${ }^{12}$ Second, the equilibrium condition in equation (3) is similar to a single-agent censored regression, except that a subvector of its regressors now consists of equilibrium objects $\left\{\mathbb{E}_{\varepsilon_{j}}\left[K_{j}^{*} \mid x, \varepsilon_{i}\right]: j \neq i\right\}$. Thus, the model lends itself to a standard maximum likelihood estimation of censored regressions with generated regressors.

We conclude this section with further justifications of our modeling choices, citing distinctive institutional features of the dialysis market. First, providers' choices of capacity are essentially continuous. Our data show that such capacity choices for a provider in a market could range from 0 to almost 60 . With such a large action space, it is practically infeasible to apply a typical multinomial choice framework to analyze capacity decisions.

Second, a dialysis provider rarely adjusts its capacity after its initial entry (see Grieco \& McDevitt, 2014). This indicates that providers' market entry decisions are practically made simultaneously with their continuous choices of capacity. Both decisions are de facto permanent, based on providers' expectation about market profitability.

Finally, providers interact through a simultaneous game with incomplete information, because each dialysis provider

\footnotetext{
12 The scale parameter $a_{i}$ could be identified if additional data are available. For instance, if the average per unit variable profits for each provider are reported in the data, then they can be used to jointly identify the scale parameter along with other parameters in the profit functions.
} 
has little information about components that affect its competitors' profits (e.g., randomness in their nephrologists' referral patterns and idiosyncratic elements in their operating and maintenance costs). Hence a provider's payoff depends on idiosyncratic information that is not known to others. An alternative assumption of complete information would have ignored such idiosyncrasies in a provider's private information set.

\section{Econometric Methods}

We now discuss the identification of coefficients $\beta_{i}, \gamma_{i, j}, b_{i}$ in equation (3) using a typical two-step argument. First, under the assumption that private information is independent across players conditional on market characteristics, player $i$ 's expectation for competitor $j$ 's capacity in equation (3) is a function of market characteristics $X$ alone. ${ }^{13}$ Provided that data are rationalized by a single profile of PBNE strategies $\left\{K_{i}^{*}: i \in N\right\}$, this function is identifiable as the expectation of $K_{j}^{*}$ conditional on $X$. With the scale normalization $a_{i}=$ $1 / 2$, equation (3) becomes

$$
K_{i}^{*}\left(X, \varepsilon_{i}\right)=\max \left\{0, X \beta_{i}+\sum_{j \neq i} \gamma_{i, j} \varphi_{j}(X)-b_{i}-\varepsilon_{i}\right\}
$$

for all $i$, where $\varphi_{j}(X) \equiv \mathbb{E}_{\varepsilon_{j}}\left[K_{j}^{*}\left(X, \varepsilon_{j}\right) \mid X\right]$ is directly identifiable if the data are generated from a single PBNE. With the distribution of $\varepsilon_{i}$ parameterized (e.g., as a normal or a logistic distribution), the joint identification of $\beta_{i}, \gamma_{i, j}, b_{i}$ and parameters in the distribution of $\varepsilon_{i}$ follows from typical arguments for parametric tobit models as long as the vector of $X$ and $\left\{\varphi_{j}(X)\right\}_{j \neq i}$ under equilibrium demonstrates sufficient variation (i.e., their joint support satisfies a typical mild full-rank condition). ${ }^{14}$

This identification strategy leads to the following twostep estimator. In the first step, estimate the expectation of providers' equilibrium capacity choices $\hat{\varphi}_{j}(X)$. This could be done using either kernel estimators (with the local constant or the polynomial approach) or sieves estimators with polynomial bases. We adopt the latter approach for the estimates reported in section VI, that is,

$$
\hat{\varphi}_{j}\left(x_{g}\right) \equiv \min _{\left\{\alpha_{s}\right\}_{0 \leq s \leq S}} \frac{1}{G} \sum_{g=1}^{G}\left[k_{g, j}-\sum_{s=0}^{S} \alpha_{s} x_{g}^{s}\right]^{2},
$$

\footnotetext{
13 In case the actual data-generating process is such that the firms' private information is positively (or negatively) correlated, our estimates may understate (or overstate) the negative effects of competitors' capacity choices on a provider's profits.

${ }^{14}$ In fact, the identification of coefficients $\beta_{i}, \gamma_{i, j}, b_{i}$ may be shown for equation (4) under nonparametric stochastic restrictions on $\varepsilon_{i}$ instead of parametric assumptions. Examples of these stochastic restrictions include independence between $\varepsilon_{i}$ and $X$ as in Buckley and James (1979) and Horowitz (1986), conditional symmetry as in Powell (1986), and median independence in Powell (1984).
}

where $g$ is an index for the $G$ independent games (markets) observed in the data and $k_{g}, j, x_{g}$ are realizations of $K_{j}, X$ in market $g$. We set the order $S$ to 4 in estimation. Alternatively, to reduce computational costs in estimation, one may choose to replace these first-step nonparametric estimates with those from a reduced-form Poisson regression. A Poisson regression works well in practice when the shapes of the empirical distribution of dependent variables are close to some distributions from the exponential family. (See Cameron \& Trivedi, 1998, and Christensen, 1997, for details.) In such cases, Poisson regressions are known to provide good approximations in terms of model fit, especially when the dependent variables are continuous or count data, as is the case in our application.

In the second step, use a maximum likelihood estimator where the distribution of $\varepsilon_{i}$ is parameterized (e.g., $\varepsilon_{i} \sim N\left(0, \sigma_{i}^{2}\right)$ for all $i$, where $\sigma_{i}^{2}$ is a parameter to be estimated). Specifically, let $\theta \equiv\left(\theta_{i}\right)_{i \in N}$ where $\theta_{i} \equiv\left(\beta_{i},\left\{\gamma_{i, j}\right\}_{j \neq i}, b_{i}, \sigma_{i}\right)$. Our two-stage estimator is defined as

$$
\hat{\theta}^{T S} \equiv \arg \max _{\theta} \frac{1}{G} \log \hat{L}_{G}(\theta)
$$

where

$$
\begin{aligned}
& \hat{L}_{G}(\theta) \equiv \prod_{g \leq G} \prod_{i \in N} \hat{f}_{i}\left(k_{g, i} \mid x_{g} ; \theta_{i}\right) ; \text { and } \\
& \hat{f}_{i}\left(k_{i} \mid x ; \theta_{i}\right) \\
& \equiv\left\{1-\Phi\left(\frac{x \beta_{i}+\sum_{j \neq i} \gamma_{i, j} \hat{\varphi}_{j}(x)-b_{i}}{\sigma_{i}}\right)\right\}^{1\left(k_{i}=0\right)} \\
& \quad \times\left\{\frac{1}{\sigma_{i}} \phi\left(\frac{k_{i}-x \beta_{i}-\sum_{j \neq i} \gamma_{i, j} \hat{\varphi}_{j}(x)+b_{i}}{\sigma_{i}}\right)\right\}^{1\left(k_{i}>0\right)}
\end{aligned}
$$

With the number of bases used in the first-step polynomial estimation expanding at an appropriate rate as the sample size increases, the preliminary estimate $\hat{\varphi}_{j}($.$) converges to the true$ function uniformly at a rate fast enough to maintain the root$n$ asymptotic normality of the MLE estimators in the second step.

Alternatively, one could also use another likelihood-based estimator, denoted $\hat{\theta}^{F L}$, which adopts a full nested fixed-point maximum-likelihood approach. This estimator amounts to replacing the first-step estimates $\hat{\varphi}_{i}$ in the two-stage estimator $\hat{\theta}^{T S}$ by $\tilde{\varphi}_{i}(x ; \theta)$, which is defined as the solution for $\left\{\varphi_{i}(x)\right\}_{i \in N}$ in the following fixed-point equation: for all $i \in N$,

$$
\begin{aligned}
& \varphi_{i}(X) \equiv \mathbb{E}_{\varepsilon_{i}}\left[K_{i}^{*}\left(X, \varepsilon_{i}\right) \mid X\right] \\
& =\mathbb{E}_{\varepsilon_{i}}\left[\max \left\{0, X \beta_{i}+\sum_{j \neq i} \gamma_{i, j} \varphi_{j}(X)-b_{i}-\varepsilon_{i}\right\} \mid X\right]
\end{aligned}
$$


Such an estimator is feasible under our specification of $\pi_{i}$ and parameterization of the private information distribution. ${ }^{15}$ The nested maximum-likelihood estimator used in our case is analogous to that applied widely to dynamic discrete choice models (Rust, 1987).

It is possible to improve efficiency in estimation using a GMM framework that further exploits the fixed-point characterization of ex ante equilibrium capacities in the second step of the two-stage estimator above. To this end, we propose a third GMM estimator that incorporates the structural fixed-point equation (8), which characterizes the expected capacity for each provider in a PBNE. More specifically, the GMM estimator is

$$
\hat{\theta}^{G M} \equiv \arg \min _{\theta} \hat{M}_{G}^{\prime}(\theta) \hat{W}_{G} \hat{M}_{G}(\theta),
$$

where the empirical moments are

$$
\hat{M}_{G}(\theta) \equiv\left[\begin{array}{c}
\frac{1}{G} \nabla_{\theta} \log \hat{L}_{G}(\theta) \\
\frac{1}{G} \sum_{g \leq G} \sum_{i \in N}\left[\tilde{\varphi}_{i}\left(x_{g} ; \theta\right)-\hat{\varphi}_{i}\left(x_{g}\right)\right]^{2}
\end{array}\right] .
$$

$\hat{L}_{G}(\theta)$ is defined in equation (6); $\hat{W}_{G}$ is a consistent estimator for the optimal GMM weight matrix; $\hat{\varphi}_{i}(x)$ is the first-stage nonparametric estimates for expected capacities in equilibrium (e.g., a polynomial approximation defined in equation [5]); and $\tilde{\varphi}_{i}(x ; \theta)$ is a solution for $\left\{\varphi_{i}(x)\right\}_{i \in N}$ in equation (8) at $X=x$ given the vector of parameters $\theta$. Alternatively, in order to reduce computational costs, one can also choose to replace $\hat{\varphi}_{i}($.$) with the fitted values of expected capacities$ based on a reduced-form Poisson regression. We adopt this approach in our estimation later. In section VI, we first obtain initial GMM estimates by setting the weight matrix to be the identity matrix. We then estimate the optimal weight matrix $\hat{W}_{G}$ using the initial estimates and apply it to weighted GMM to improve estimation efficiency.

We conclude this section with several remarks regarding the issue of multiple equilibria and the comparison between the three estimators $\hat{\theta}^{T S}, \hat{\theta}^{F L}$, and $\hat{\theta}^{G M}$.

Remark 1. For any given market characteristics $x$ and any vector of parameters $\theta$, there could potentially be multiple solutions for $\left\{\varphi_{i}(x)\right\}_{i \in N}$ in equation (8). However, such multiplicity does not affect the validity of our two-stage estimator under the common assumption that the data are rationalized by a single Bayesian Nash equilibrium (BNE). This is because under this single equilibrium assumption, a provider's expected capacity in the likelihood is directly identified from the data rather than solved for from equation (8).

$$
\begin{aligned}
& { }^{15} \text { Under the normality assumption of } \varepsilon \text {, either (8) has a closed form, } \\
& \varphi_{i}=\Phi\left(\frac{x \beta_{i}+\sum_{j \neq i} \gamma_{i, j} \varphi_{j}-b_{i}}{\sigma_{i}}\right) \\
& \quad \times\left\{x \beta_{i}+\sum_{j \neq i} \gamma_{i, j} \varphi_{j}-b_{i}+\sigma_{i} \frac{\phi\left(\frac{x \beta_{i}+\sum_{j \neq i} \gamma_{i, j} \varphi_{j}-b_{i}}{\sigma_{i}}\right)}{\Phi\left(\frac{x \beta_{i}+\sum_{j \neq i} \gamma_{i, j} \varphi_{j}-b_{i}}{\sigma_{i}}\right)}\right\},
\end{aligned}
$$

for $i \in N$, where $\varphi_{i}$ is the expectation of provider $i$ 's capacity in equilibrium.
On the other hand, the full nested fixed-point MLE estimator is susceptible to the issue of multiple equilibria. To deal with this, we do not directly apply the original nested fixed-point MLE, but instead adopt an alternative approach proposed by Judd and Su (2012). This approach, known as the mathematical program with equilibrium constraints (MPEC), reformulates the original problem into the maximization of likelihood over the space of structural parameters and strategies subject to a set of constraints that define PBNE. Hence it avoids the need to solve for the inner loop in the full nested fixed-point MLE. The MPEC algorithm implicitly deals with the multiplicity issue through an effective ad hoc procedure, which always picks an equilibrium that maximizes the likelihood. (More detailed discussions are included in section V.C).

Remark 2. The multiplicity also does not affect the validity (consistency) of our GMM estimator under the assumption of a single BNE in the data, as we have incorporated the following ad hoc procedure in the calculation of $\hat{\theta}^{G M}$. Suppose for some $\left(x_{g}, \theta\right)$ the system of equations in equation (8) admits multiple solutions of the vector $\tilde{\varphi}\left(x_{g} ; \theta\right) \equiv\left\{\tilde{\varphi}_{i}\left(x_{g} ; \theta\right)\right\}_{i \in N}$ (which are often picked up by experimenting with multiple initial points while solving the nonlinear fixed-point equation in that equation). In such cases, choose the vector of $\tilde{\varphi}\left(x_{g} ; \theta\right)$ that minimizes the empirical moments in equation (10) while evaluating the objective function of GMM. To see how such a procedure maintains the consistency of $\hat{\theta}^{G M}$ under multiple equilibria, note that the second set of moments in section (10) takes a form similar to the objective function of a minimum-distance estimator. Thus, this procedure is effectively using the directly identifiable $\mathbb{E}\left[K_{i}^{*}\left(X, \varepsilon_{i}\right) \mid X\right]$ to guide our choices of equilibrium-implied expected capacity while implementing GMM.

Remark 3. The two-stage estimator $\hat{\theta}^{T S}$ and the full nested fixed-point ML estimator $\hat{\theta}^{F L}$ have respective advantages. As explained in remark $1, \hat{\theta}^{T S}$ is robust to the issue of multiple equilibria but does not explicitly use the structure in the fixed-point characterization of ex ante capacities in equilibrium (i.e., the $\varphi_{i}$ 's). In contrast, $\hat{\theta}^{F L}$ is explicit in exploiting this structural relation defining $\left\{\varphi_{i}\right\}_{i \in N}$, but is potentially susceptible to the issue of multiple equilibria. ${ }^{16}$ Therefore, choices between the two should depend on researchers' judgment about the possibility of multiple equilibria in the data-generating process. The GMM estimator $\hat{\theta}^{G M}$ provides the benefits of both estimators. Due to the use of the second set of moments, it not only exploits the structural relations defining ex ante capacity but also manages to deal with the issue of equilibrium multiplicity under the assumption that choices of capacity in the data are rationalized by a single pure-strategy Bayesian Nash equilibrium.

\footnotetext{
16 The full-maximum-likelihood estimator should be more efficient than the two-step estimator, provided the identification of parameters holds under the parameterization, and the model always admits a unique PBNE under all $x$ and $\theta$.
} 


\section{Data Description}

\section{A. Construction of the Sample and the Definition of Markets}

We construct our sample from the Dialysis Facility Compare Data published by the Center for Medicare and Medicaid Services (CMS) in 2007. The CMS receives monthly updates about the characteristics of each facility (e.g., name, address, chain affiliation, number of dialysis stations, date of certification) and posts them online every quarter. Several recent studies on the dialysis market have used a similar data set (e.g., Ramanarayanan \& Snyder, 2011; Grieco \& McDevitt, 2014; Cutler et al., 2012). The key variable of interest is a provider's choice of capacity, or the number of dialysis stations.

The market for outpatient dialysis is local in nature. Dialysis patients usually receive three treatment sessions per week, each of which lasts for about four hours. They are in general unwilling (or unable) to travel too far. According to the Medicare Payment Advisory Commission (MedPAC), the median driving distance between patients and dialysis facility is 6 miles. Following several other studies on dialysis markets (Grieco \& McDevitt, 2014; Cutler et al., 2012), we use Hospital Service Area (HSA) to delineate the local market. HSA is compiled by the Dartmouth Atlas (Dartmouth Medical School, 2006) from Medicare data on patients' choices of hospitals. Such an area is relatively self-contained with respect to health care services. ${ }^{17}$ The number of HSAs in the United States is roughly equal to the number of U.S. counties; however, their boundaries do not overlap in general. Unfortunately, demographics such as population, age, and racial composition are not available at the HSA level. To obtain the market-level profit and the cost shifters, we assign each HSA to a county based on the population distribution within the HSA. ${ }^{18}$ Then we use county-level demographics data from the U.S. Census (e.g., racial composition, age, income, poverty, size of business payroll) to approximate the population characteristics within an HSA.

We supplement the demographics data from the Census with hospital and physician capacity data from the Dartmouth Atlas (Dartmouth Medical School, 2006). While the Dartmouth Atlas reports this information only for 2006, it provides a good approximation for 2007 because there were no major shifts in the industry environment between these two consecutive years. We use the age-adjusted prevalence rate of diabetes as a proxy for ESRD risks. We also use hospital beds and the number of nephrologists to control for the base demand and the intensity of health care. We include these variables because market population alone does not

\footnotetext{
${ }^{17}$ Specifically, an HSA is defined by assigning postal codes to the hospital area where the greatest proportion of their Medicare residents (including those with ESRD) were hospitalized. In the absence of detailed patientlevel information, HSA approximates geographic markets for dialysis more closely than alternative definitions (e.g., county, state, or metropolitan statistical area).

18 We decompose each HSA into a collection of postal codes and obtain the population for each code from the Census. We assign HSA to a county if that county contains the largest proportion of HSA population.
}

accurately reflect the size of the customer base relevant to the dialysis service industry. By including the diabetic prevalence rates and other factors related to ERSD risks, we expect to obtain a better measure of the size of the customer base for dialysis treatment in each market. Hence our treatment of the market size differs slightly from the classical approach in entry games such as in Bresnahan and Reiss (1991), but is comparable to those in Jia (2008) and Ciliberto and Tamer (2009). Moreover, our estimation results that follow show these additional variables explain a significant portion of the variation in the capacity choice of dialysis providers.

Following Ford and Kaserman (1993), who showed the certificate of need (CON) regulation, discouraged entry by requiring additional regulatory procedure for providers to establish their market presence, we construct a binary indicator for the state-level CON regulation. Finally, we use the distance between an HSA and the headquarters of the chains as cost shifters. ${ }^{19}$

The distribution of dialysis capacity is highly skewed. For example, in a sample that includes almost all HSAs in the lower 48 states, the average market capacity in a given market is about 21, but the total dialysis capacity in some markets can be as high as 1,309. The high-capacity outliers usually lie in heavily populated cities such as Chicago and Los Angeles. In these markets, chain providers often operate multiple branches in close proximity to each other. The nature of competitive interactions is clearly different from an average market. For our analysis, we chose to focus on areas with population between 40,000 and 800,000.20 We exclude another 124 outliers with total market capacity greater than $60 .{ }^{21}$ As a robustness check, we perform the analysis in larger samples that include almost all HSAs using the two-stage estimator. We obtain results very similar to what we report in the paper. These additional results are reported in panel A of table A1 in the appendix. Our main results are also robust to alternative sample selection criteria as shown in panels B and C in table A 1.22

19 We use the HSA boundary file from Dartmouth Atlas (Dartmouth Medical School, 2006) to pinpoint the centroid for the market. The distance from the geographical center of each HSA to the headquarters of either chain is calculated using the Haversine formula.

20 This eliminates 1,646 HSAs, most of them sparsely populated rural areas and quite different from an average market. According to MedPAC, the median distance between a rural patient and a dialysis station is almost four times longer than for urban patients. Therefore, patients in rural areas are more likely to seek outside options such as hospital units or home dialysis service. Nevertheless, we test the robustness of our results in panel A of table A1 using an alternative sample selection criterion based on an enlarged sample with more than 3,000 HSAs and get results very similar to our main specification. Besides, our sample selection criterion is in line with other studies (e.g., Bresnahan \& Reiss, 1991; Collard-Wexler, 2013).

21 Our current cutoff is approximately the 90th percentile in the distribution of total capacity in a market. Our results are robust to alternative cutoff values (e.g., the 95th or the 85th percentile in capacity distribution). See panels B and $\mathrm{C}$ in table A1 for details.

22 There is no obvious evidence that the actual geographic market should be more disaggregated than HSA. If the actual market is more disaggregated than HSA, we would obtain very different results when large markets are excluded from the estimation sample. In an additional robustness check, we perform our analysis using the two-stage estimator after excluding market in areas with more than 200,000 residents. The estimates obtained using the smaller sample are similar to our main specification. 
TABlE 1.-Summary Statistics of CAPACity Distribution

\begin{tabular}{|c|c|c|c|c|c|}
\hline Variable & Definition & Mean & $\mathrm{SD}$ & Minimum & Maximum \\
\hline$K_{f m c}$ & FMC's capacity decision & 6.40 & 11.4 & 0 & 60 \\
\hline$K_{d a v}$ & DaVita's capacity decision & 4.40 & 9.22 & 0 & 54 \\
\hline$K_{\text {nonchain }}$ & Nonchain's capacity decision & 5.32 & 10.0 & 0 & 58 \\
\hline$I_{f m c}$ & $I\left(K_{f m c}>0\right)$ & 0.31 & 0.46 & 0 & 1 \\
\hline$I_{d a v}$ & $I\left(K_{d a v}>0\right)$ & 0.23 & 0.42 & 0 & 1 \\
\hline$I_{\text {nonchain }}$ & $I\left(K_{\text {nonchain }}>0\right)$ & 0.28 & 0.45 & 0 & 1 \\
\hline$K_{f m c} \mid I_{f m c}=1$ & FMC's capacity conditional on entry & 20.4 & 11.2 & 2 & 60 \\
\hline$K_{d a v} \mid I_{d a v}=1$ & DaVita's capacity conditional on entry & 19.3 & 9.24 & 7 & 54 \\
\hline$K_{\text {nonchain }} \mid I_{\text {nonchain }}=1$ & Nonchain's capacity conditional on entry & 18.9 & 10.0 & 1 & 58 \\
\hline
\end{tabular}

Our final sample contains 1,320 HSA and 1,287 facilities in the 48 contiguous states in 2007. Conditional on entry into these markets, chain dialysis providers usually open a single branch. One potential concern is that capacity decisions of chain providers are correlated across markets. However, such a concern is minimal for our analysis since our sample selection criterion ensures the markets are relatively isolated. A back-of-the-envelope spatial analysis shows that the average distance from an FMC facility in the sample to its closest FMC neighbor is 12.5 miles; the same statistic for DaVita is 12.3 miles.23 Most of the facilities (287 out of 353 for DaVita and 242 out of 490 for FMC) are not within 10 miles of another facility of the same chain. Overall, there is no evidence that correlation between capacity decisions should be a concern for our analysis.

\section{B. Descriptive Statistics}

Table 1 summarizes the capacity choices of FMC, DaVita, and nonchain providers. There are two significant empirical regularities. First, dialysis providers' capacity choices vary substantially over a wide support. For example, the average capacity of FMC is 6.4, followed by nonchain and DaVita. The standard deviations for these providers range between 9.22 and 11.4. While one may estimate a multinomial choice model by grouping capacity levels into aggregate categories (such as low, medium, and high capacity), this type of exercise would overlook the information contained in the rich variation in the choices of capacity. Furthermore, in our sample, the number of dialysis stations in a given facility ranges from 1 to 54. Thus, even after removing the outliers, the action space remains too large for a multinomial choice model. Therefore, we analyze dialysis providers' capacity decisions as continuous choices. Second, there is a substantial number of markets in which some providers choose not to enter. FMC, the largest provider, enters about $31 \%$ of the markets (followed by nonchain, 28\%, and DaVita, 23\%). Thus, the overall unconditional expectation of capacity is much smaller than the mean capacity conditional on entry.

The correlation between entry and capacity decisions in table 2 presents descriptive evidence for strategic interactions. A provider's market presence and capacity choice are

\footnotetext{
${ }^{23}$ The calculation of distances is based on both in-sample and out-ofsample facilities.
}

TABle 2.-CAPACity CoRrelations

\begin{tabular}{lrrrrrr}
\hline \hline & \multicolumn{6}{c}{ Correlation } \\
\cline { 2 - 6 } & \multicolumn{1}{c}{$I_{\text {fmc }}$} & $I_{\text {dav }}$ & $I_{\text {nonchain }}$ & $K_{\text {fmc }}$ & $K_{\text {dav }}$ & $K_{\text {nonchain }}$ \\
\hline$I_{\text {fmc }}$ & 1 & & & & & \\
$I_{\text {dav }}$ & -0.12 & 1 & & & & \\
$I_{\text {nonchain }}$ & -0.13 & -0.07 & 1 & & & \\
$K_{\text {fmc }}$ & 0.83 & -0.12 & -0.12 & 1 & & \\
$K_{\text {dav }}$ & -0.14 & 0.88 & -0.04 & -0.13 & 1 & \\
$K_{\text {nonchain }}$ & -0.13 & -0.09 & 0.85 & -0.13 & -0.06 & 1 \\
\hline Number of observations: $1,320$. & & & &
\end{tabular}

both negatively correlated with its competitors'. For example, the correlation between FMC and DaVita's entry decisions is -0.12 , while the correlation between DaVita's capacity and FMC's entry decision is -0.14 . This suggests DaVita and FMC generally enter different markets, and DaVita is even less likely to enter when FMC chooses a larger capacity.

However, we cannot infer from these aggregate correlation patterns alone that all providers' response curves to competitors' choices of capacity are downward sloping, for this would risk overlooking the heterogeneity in providers' profit and cost structures. The observed aggregate correlation pattern in table 2 could be mostly driven by a subset of providers or a subset of markets in data and thus may not be representative of the other providers or markets in general. Indeed, a key motivation for our structural analyses in section IV is the need to account for such heterogeneity in the reaction curve across providers.

Table 3 presents summary statistics of the market demographics. An average market in our sample is populated by 75,531 residents, 1,030 miles away from FMC's headquarter and 1,100 miles away from Davita's headquarter. About $23 \%$ of the markets are located in the Northeast region, $24 \%$ in the Midwest, $17 \%$ in the West, and the remaining $31 \%$ in the South. The CON regulation is effective in $21 \%$ of the markets. We employ a parsimonious set of profit and cost shifters, including the percent of population over age 65 and racial composition. In alternative specifications, we experiment with a larger set of variables such as poverty rate, income, population density, size of business payroll, number of uninsured, number of hospital registered nurses, and size and racial mixes of Medicare enrollees. These variables do little to explain the variation in dialysis capacity, and therefore we did not include them in our main specification. 
Table 3.-Summary Statistics of Market Variables

\begin{tabular}{|c|c|c|c|}
\hline Variable & Definition & Mean & SD \\
\hline pop* & Total HSA population from DA & 75,531 & 65,734 \\
\hline black & Percent black from Census & 0.07 & 0.09 \\
\hline white & Percent white from Census & 0.88 & 0.11 \\
\hline latino & Percent Latino from Census & 0.09 & 0.11 \\
\hline asian & Percent Asia from Census & 0.02 & 0.03 \\
\hline age1 & $\begin{array}{l}\text { Percent population age between } 22 \text { and } \\
44 \text { from Census }\end{array}$ & 0.33 & 0.04 \\
\hline age 2 & $\begin{array}{l}\text { Percent population age between } 44 \text { and } \\
65 \text { from Census }\end{array}$ & 0.34 & 0.04 \\
\hline age 3 & Percent population age $65+$ from Census & 0.14 & 0.03 \\
\hline neph & $\begin{array}{l}\text { Number of nephrologists per } 1,000 \\
\text { population from DA }\end{array}$ & 1.51 & 1.00 \\
\hline bed* & $\begin{array}{l}\text { Number of hospital bed per } 1,000 \\
\text { population from DA }\end{array}$ & 2.59 & 0.89 \\
\hline rn & $\begin{array}{l}\text { Number of registered nurse per } 1,000 \\
\text { population from DA }\end{array}$ & 1.32 & 0.27 \\
\hline dbrate & Prevalence rate of diabetes & 8.42 & 1.63 \\
\hline conreg & CON regulation indicator & 0.21 & 0.41 \\
\hline $\mathrm{NE}$ & Northeast region indicator & 0.23 & 0.42 \\
\hline MW & Midwest region indicator & 0.24 & 0.42 \\
\hline West & West region indicator & 0.17 & 0.37 \\
\hline dfmc & $\begin{array}{l}\text { Distance to FMC's headquarter in } 1,000 \\
\text { miles }\end{array}$ & 1.03 & 0.73 \\
\hline dfmc2 & dfmc squared & 1.60 & 2.04 \\
\hline ddav & $\begin{array}{l}\text { Distance to DaVita's headquarter in } \\
1,000 \text { miles }\end{array}$ & 1.10 & 0.38 \\
\hline ddav2 & ddave squared & 1.34 & 0.83 \\
\hline
\end{tabular}

Number of observations: 1,320. Variables labeled with an asterisk enter the estimation in logs and are reported without logging in this table. Explanatory variables come from the Dartmouth Atlas (Dartmout Medical School, 2006) Census Bureau when indicated and otherwise are constructed by the authors.

\section{Details in Implementing the Estimators}

We estimate the model using the estimators described in section IV. Table 4 presents the results. Panels A, B, and C present the results from the two-stage estimator $\hat{\theta}^{T S}$, the maximum likelihood (nested fixed-point) estimator $\hat{\theta}^{F L}$, and the GMM estimator $\hat{\theta}^{G M}$, respectively. We now provide further details in the implementation of these estimators.

In the first step of the two-stage estimation, we adopt the Poisson regression approach to estimate each provider's expected capacity in equilibrium. That is, we fit the observed capacity choices to a Poisson distribution and use it to estimate a provider's expected capacity. A Poisson regression is convenient because the observed capacity choices are nonnegative, with many observations censored at 0 . In a finite sample with a moderate size such as ours, a Poisson distribution fits the data better than the nonparametric alternative of polynomial approximation. ${ }^{24}$ In the second step, we use a tobit model to estimate the effect of $\widehat{\mathbb{E}}\left(K_{-i}^{*} \mid X\right)$ and $X$ on $K_{i}$, where $\widehat{\mathbb{E}}\left(K_{-i}^{*} \mid X\right)$ is the predicted capacity obtained from the first step. We follow a standard bootstrap procedure to calculate the standard errors of the estimates, based on 300 bootstrap samples.

We estimate $\hat{\theta}^{F L}$ by reformulating the nested fixed-point MLE as the MPEC optimization strategy introduced by Judd and Su (2012). The standard maximization routines for

${ }^{24}$ The $R^{2}$ in Poisson regression are $31 \%, 30 \%$, and $23 \%$ for FMC, DaVita, and nonchain; they are $22 \%, 22 \%$, and $18 \%$ in polynomial approximation.
TABle 4.-STRategic CAPaCity Model

\begin{tabular}{|c|c|c|c|c|c|c|}
\hline & \multicolumn{2}{|c|}{ FMC } & \multicolumn{2}{|c|}{ Dav } & \multicolumn{2}{|c|}{ Nonchain } \\
\hline & EST & SE & EST & SE & EST & SE \\
\hline & \multicolumn{6}{|c|}{ A. Two-Stage Estimation $\left(\hat{\theta}^{T S}\right)^{\mathrm{a}}$} \\
\hline $\mathrm{K}_{f m c}$ & & & -1.06 & $0.24^{* * *}$ & -0.73 & $0.24^{* * *}$ \\
\hline $\mathrm{K}_{d a v}$ & -1.07 & $0.26^{* * *}$ & & & -0.68 & $0.26^{* * *}$ \\
\hline $\mathrm{K}_{\text {nonchain }}$ & -1.43 & $0.26^{* * *}$ & -1.04 & $0.30^{* * *}$ & & \\
\hline lpop & 25.0 & $2.20^{* * *}$ & 24.9 & $2.43^{* * *}$ & 21.7 & $2.10^{\text {*** }}$ \\
\hline neph & 2.46 & $0.76^{* * *}$ & 3.38 & $0.99^{* * *}$ & 1.23 & 0.87 \\
\hline lbed & 1.39 & 4.19 & -5.34 & 4.51 & 4.04 & 4.65 \\
\hline $\mathrm{rn}$ & 9.87 & $-4.45^{* *}$ & 11.3 & $4.84^{* *}$ & 3.44 & 5.04 \\
\hline dbrate & 2.21 & $0.76^{* * *}$ & 1.35 & 0.93 & 0.44 & 0.84 \\
\hline conreg & -4.84 & $1.92^{* *}$ & -2.66 & 1.99 & -3.54 & $2.11^{*}$ \\
\hline $\mathrm{NE}$ & -8.16 & $4.04^{* *}$ & -10.0 & $5.07^{* *}$ & 7.68 & 4.99 \\
\hline MW & -8.80 & $2.54^{* * *}$ & -4.71 & 3.26 & -5.35 & $2.96^{*}$ \\
\hline West & -8.49 & $4.00^{* *}$ & -10.8 & $5.18^{* *}$ & 10.5 & $4.10^{* *}$ \\
\hline dfmc & 10.9 & 9.18 & 30.7 & $10.7^{* * *}$ & 33.8 & $11.7^{* * *}$ \\
\hline $\mathrm{dfmc} 2$ & -3.96 & 3.03 & -8.22 & $3.69^{* *}$ & -12.3 & $3.65^{* * *}$ \\
\hline ddav & 12.6 & 14.2 & 7.70 & 16.9 & 72.9 & $13.6^{* * *}$ \\
\hline ddav2 & -6.27 & 7.12 & 2.99 & 8.38 & -33.3 & $6.85^{* * *}$ \\
\hline black & 60.2 & $22.6^{* * *}$ & 22.5 & 22.5 & 81.0 & $44.2^{*}$ \\
\hline white & 22.8 & 20.7 & -14.1 & 20.3 & 69.2 & 43.3 \\
\hline latino & 38.1 & $8.56^{* * *}$ & 7.69 & 10.3 & 16.1 & 10.2 \\
\hline asian & -86.6 & $50.1^{*}$ & 21.5 & 47.5 & 87.9 & 56.4 \\
\hline age 1 & 29.0 & 45.9 & -98.2 & $52.7^{*}$ & -12.0 & 47.8 \\
\hline age2 & 81.9 & $39.7^{* *}$ & -40.7 & 46.8 & 7.13 & 42.9 \\
\hline age3 & 53.4 & 37.0 & -45.7 & 43.9 & 28.2 & 41.3 \\
\hline cons & -384 & $45.6^{* * *}$ & -270 & $55.0^{* * *}$ & -382 & $61.0^{* * *}$ \\
\hline sigma & 23.0 & $0.71^{* * *}$ & 24.1 & $0.86^{* * *}$ & 22.9 & $0.74^{* * *}$ \\
\hline & \multicolumn{6}{|c|}{ B. Fixed Point ML $\left(\hat{\theta}^{F L}\right)^{\mathrm{b}}$} \\
\hline $\mathrm{K}_{f m c}$ & & & -1.88 & $0.19^{* * *}$ & & \\
\hline $\mathrm{K}_{d a v}$ & -2.32 & $0.25^{* * *}$ & & & -1.86 & $0.22^{* * *}$ \\
\hline $\mathrm{K}_{\text {nonchain }}$ & -1.44 & $0.20^{* * *}$ & -1.73 & $0.26^{* * *}$ & -1.58 & $0.23^{* * *}$ \\
\hline lpop & 25.0 & $1.59^{* * *}$ & 28.3 & $2.23^{* * *}$ & 26.9 & $2.11^{* * * *}$ \\
\hline neph & 2.11 & $0.76^{* * *}$ & 2.34 & $0.89^{* * *}$ & 2.11 & $0.93^{* *}$ \\
\hline lbed & -0.37 & 4.00 & -0.55 & 4.80 & -0.04 & 4.63 \\
\hline $\mathrm{rn}$ & 10.8 & $4.33^{* * *}$ & 12.1 & $5.12^{* * *}$ & 10.7 & $4.95^{* *}$ \\
\hline dbrate & 1.47 & $0.76^{* *}$ & 1.47 & $0.92^{*}$ & 0.91 & 0.91 \\
\hline conreg & -3.93 & $1.90^{* *}$ & -3.91 & $2.25^{* *}$ & -4.08 & $2.16^{* *}$ \\
\hline $\mathrm{NE}$ & -6.17 & $-4.03^{*}$ & -5.38 & 4.92 & -0.66 & 4.70 \\
\hline MW & -7.35 & $2.61^{* * *}$ & -8.09 & $3.18^{* * *}$ & -7.65 & $3.09^{* * *}$ \\
\hline West & 4.78 & 4.35 & 8.54 & $5.55^{*}$ & 12.7 & $4.64^{* * *}$ \\
\hline $\mathrm{dfmc}$ & 22.7 & $10.1^{* *}$ & 30.4 & $12.1^{* * *}$ & 37.8 & $11.4^{* * *}$ \\
\hline dfmc2 & -8.91 & $3.30^{* * *}$ & -11.6 & $3.98^{* * *}$ & -14.4 & $3.60^{* * *}$ \\
\hline ddav & 2.51 & 13.3 & 10.7 & 16.0 & 32.6 & $15.8^{* *}$ \\
\hline ddav2 & -0.73 & 6.37 & -3.74 & 7.69 & -13.6 & $7.47^{* *}$ \\
\hline black & 41.8 & $19.4^{* *}$ & 43.2 & $20.4^{* *}$ & 65.9 & $32.2^{* *}$ \\
\hline white & 15.6 & 18.3 & 15.8 & 18.4 & 42.4 & $30.9^{*}$ \\
\hline latino & 14.7 & $8.65^{* *}$ & 13.5 & 11.2 & 12.6 & 10.1 \\
\hline asian & 25.3 & 42.3 & 40.7 & 43.8 & 64.8 & $49.2^{*}$ \\
\hline age 1 & -7.01 & 46.3 & -18.4 & 53.3 & -9.17 & 50.4 \\
\hline age2 & -3.77 & 40.3 & -16.7 & 45.7 & -18.8 & 44.0 \\
\hline age3 & 90.4 & $39.7^{* *}$ & 90.7 & $47.9^{* *}$ & 86.2 & $44.9^{* *}$ \\
\hline cons & -330 & $39.6^{* * *}$ & -375 & $51.4^{* * *}$ & -395 & $51.9^{* * *}$ \\
\hline sigma & 20.4 & $0.80^{* * *}$ & 22.3 & $1.15^{* * *}$ & 22.5 & $1.03^{* * *}$ \\
\hline \multicolumn{7}{|c|}{$\mathrm{C}: \mathrm{GMM}\left(\hat{\theta}^{G M}\right)^{\mathrm{c}}$} \\
\hline $\mathrm{K}_{f m c}$ & & & -2.17 & $0.09^{* * *}$ & -0.82 & $0.10^{* * *}$ \\
\hline $\mathrm{K}_{d a v}$ & -0.76 & $0.10^{* * *}$ & & & -0.61 & $0.08^{* * *}$ \\
\hline $\mathrm{K}_{\text {nonchain }}$ & -0.86 & $0.17^{* * *}$ & -1.45 & $0.16^{* * *}$ & & \\
\hline lpop & 20.1 & $1.22^{* * *}$ & 35.1 & $1.19^{* * *}$ & 20.9 & $0.78^{* * *}$ \\
\hline neph & 2.00 & $0.11^{* * *}$ & 4.36 & $0.11^{* * *}$ & 2.00 & $0.05^{* * *}$ \\
\hline lbed & 3.86 & $0.97^{* * *}$ & 4.74 & $0.80^{* * *}$ & 1.06 & 0.89 \\
\hline rn & 6.39 & $2.08^{* * *}$ & 9.59 & $1.80^{* * *}$ & 5.35 & $0.78^{* * *}$ \\
\hline dbrate & 2.03 & $0.68^{* * *}$ & 2.43 & $0.55^{* * *}$ & 0.51 & 0.67 \\
\hline conreg & -4.08 & $0.42^{* * *}$ & -5.10 & $0.38^{* * *}$ & -2.60 & $0.15^{* * *}$ \\
\hline $\mathrm{NE}$ & -9.45 & $3.13^{* * *}$ & -16.5 & $2.88^{* * *}$ & 5.70 & $0.98^{* * *}$ \\
\hline MW & -6.74 & $0.98^{* * *}$ & -8.54 & $0.87^{* * *}$ & -6.56 & $0.36^{* * *}$ \\
\hline West & -7.24 & $0.88^{* * *}$ & -13.4 & $0.78^{* * *}$ & 9.10 & $1.23^{* * *}$ \\
\hline dfmc & -1.41 & 6.10 & 7.91 & $5.66^{*}$ & 28.3 & $2.93^{* * *}$ \\
\hline dfmc2 & -0.02 & 1.75 & -1.17 & 1.60 & -10.7 & $0.89^{* * *}$ \\
\hline
\end{tabular}




\begin{tabular}{|c|c|c|c|c|c|c|}
\hline & \multicolumn{2}{|c|}{ FMC } & \multicolumn{2}{|c|}{ Dav } & \multicolumn{2}{|c|}{ Nonchain } \\
\hline & EST & SE & EST & SE & EST & SE \\
\hline & \multicolumn{6}{|c|}{ C: GMM $\left(\hat{\theta}^{G M}\right)^{\mathrm{c}}$} \\
\hline ddav & -4.25 & 8.39 & 5.98 & 6.73 & 63.2 & $10.6^{* * *}$ \\
\hline ddav2 & 0.00 & 4.69 & -0.62 & 3.71 & -30.3 & $3.89^{* * *}$ \\
\hline black & 70.7 & $14.9^{* * *}$ & 75.6 & $9.88^{* * *}$ & 82.9 & 88.2 \\
\hline white & 28.8 & $18.8^{*}$ & 12.7 & 12.6 & 69.1 & 88.9 \\
\hline latino & 27.6 & $16.8^{*}$ & 22.7 & $13.1^{* *}$ & 16.1 & $10.3^{*}$ \\
\hline asian & -30.8 & 72.1 & -11.2 & 48.9 & 65.2 & 110 \\
\hline age1 & 12.9 & 175 & -78.8 & 138 & 20.0 & 112 \\
\hline age2 & 41.0 & 131 & -29.1 & 103 & 35.0 & 86.6 \\
\hline age3 & 44.5 & 112 & 10.9 & 88.6 & 39.4 & 74.9 \\
\hline cons & -298 & $129 * *$ & -416 & $106^{* * *}$ & -384 & $146^{* * *}$ \\
\hline sigma & 21.3 & $0.19^{* * *}$ & 25.1 & $0.04^{* * *}$ & 22.7 & $0.07^{* * *}$ \\
\hline
\end{tabular}

${ }^{a}$ The estimates are obtained using two-stage estimator. $\mathrm{K}_{f n c}, \mathrm{~K}_{d a v}, \mathrm{~K}_{\text {nonchain }}$ are the expected capacity choice of FMC, DaVita, and nonchain, respectively. The expected capacity choices of each provider are estimated in the first step using a Poisson regression, with regressors being the polynomials of marke characteristics. The standard errors are calculated using a bootstrap procedure. lpop and lbed are logged population and logged number of hospital beds. The definitions of other variables are presented in table 3 . ${ }^{\mathrm{b}}$ The estimates are obtained using maximum likelihood. $\mathrm{K}_{f m c}, \mathrm{~K}_{d a v}, \mathrm{~K}_{\text {nonchain }}$ are the expected capacity choice of FMC, DaVita, and nonchain, respectively. The nested fixed point problem is recast as MPEC and estimated using Knitro. lpop and lbed are logged population and logged number of hospital beds. The definitions of other variables are presented in table 3 .

${ }^{c}$ The estimates are obtained through GMM. $\mathrm{K}_{f m c}, \mathrm{~K}_{d a v}, \mathrm{~K}_{\text {nonchain }}$ are the expected capacity choices of FMC, DaVita, and nonchain, respectively. lpop and lbed are logged population and logged number of hospital beds. The definitions of other variables are presented in table 3 .

calculating the nested fixed-point MLE is computationally demanding, because it requires solving for equilibrium outcomes defined by the fixed-point mapping (8) in every market for every iteration of parameter values throughout the maximization routine. Besides, the issue of multiplicity arises in such routines because a given parameter may well admit more than one Bayesian Nash equilibria in general.

In comparison, the MPEC algorithm maximizes the likelihood with respect to both model parameters and providers' strategies (as characterized by each provider's expected capacity) in each market subject to the constraints that the expected capacity choices constitute an equilibrium in the model. As Judd and Su (2012) showed, the solution to the constrained maximum likelihood is equivalent to the solution of the nested fixed point. MPEC differs from nested fixed-point MLE computationally in that the constrained maximization in MPEC does not require solving for the nonlinear fixed-point equation in every market. For our application, there are 1,320 markets with three choice variables in each market. The likelihood is maximized with respect to 3,960 more parameters (in addition to the covariates of our empirical specification) subject to 3,960 constraints defined by equation (8). Besides, MPEC is also known to have dealt with the issue of multiple equilibria implicitly; that is, evaluating the likelihood at an MPEC solution is equivalent to evaluating the likelihood at a nested fixed-point MLE solution when the equilibrium selection mechanism is degenerate at an equilibrium that yields the highest likelihood. (For more details, see proposition 1 in Judd \& Su, 2012, and the subsequent discussions.) The standard errors are obtained through the Hessian of the likelihood function evaluated at our estimates.

To implement our GMM estimator, we use two sets of moment conditions as defined in equation (10). The first set consists of the first-order condition for maximizing the likelihood defined in equation (6). The second set of moments matches the expected capacity predicted in equilibrium to that directly recovered from data. To reduce computational costs, we use fitted values from a Poisson regression as the first-step estimates $\hat{\varphi}_{i}$ and use the MPEC algorithm to find the maximizer of the GMM objective function. We follow a standard sequential approach for estimation using GMM: first obtain an initial GMM estimate by setting the weight matrix to be the identity matrix and then use it to compute the optimal weight matrix. We then reestimate the model by substituting the optimal weight matrix into the GMM objective function. The standard errors for the GMM estimator $\hat{\theta}^{G M}$ are then calculated using the classical approach as in section 6 of Newey and McFadden (1994). ${ }^{25}$

\section{Results}

\section{A. Estimates}

The estimates from each panel of table 4 are close in magnitude. The standard errors for GMM estimators are often smaller than those for the two-step estimators (e.g., population, nephrologist, diabetes rate, register nurse, CON regulation), especially the standard errors on coefficients of strategic variables. This seems to suggest gains in estimation efficiency from exploiting the equilibrium structure of our model. Since GMM is advantageous over two-stage and fixed-point maximum likelihood, we focus our discussion based on GMM estimates (unless otherwise indicated).

The strategic effect of rival's capacity is strongly significant with a negative sign. This result is robust to the estimators used. The magnitudes of the strategic coefficients suggest that FMC competes aggressively with all other providers. For example, according to the GMM estimates, the effect of FMC on DaVita is about 50\% larger than the effect of nonchain $(-2.17$ versus -1.45$)$ while it is $30 \%$ larger than the effect of DaVita on nonchain $(-0.82$ versus -0.61$)$. Nevertheless, there is no significant evidence that competition is more intense between the two chain providers. The difference in the strategic effects may be ascribed to the heterogeneity in providers and market conditions. For example, different providers often have access to different networks of nephrologists that they can expect to get referrals from. Discrepancies in business strategies or management styles across providers may also explain part of the heterogeneity.

The strategic effects are quite large. A quick calculation of the marginal effects ( similar to those calculated for a tobit model) suggests that, holding other factors in the profits fixed at their mean value, a 1 unit increase in DaVita's expected capacity in equilibrium decreases FMC's capacity by 0.24

\footnotetext{
25 This classical approach essentially amounts to stacking all moments used for estimation together (i.e., including those used for estimating the linear coefficients in the Poisson regressions in the first step) and then estimating the covariance matrix based on such an augmented set of moments.
} 
units and a 1 unit increase in nonchain's expected capacity decreases FMC's capacity by 0.27 unit. For both DaVita and nonchain, FMC poses a stronger competitive pressure. A 1 unit increase in FMC's expected capacity reduces DaVita's capacity by 0.37 units and nonchain's capacity by 0.21 units.

The strategic interactions between dialysis providers also imply a strong effect of capacity choices on competitors' entry probabilities. Our estimates imply that FMC poses the strongest competition to both DaVita and nonchain. A 1 unit increase in FMC's capacity decreases the entry probability of DaVita and nonchain by 0.03 and 0.01 , respectively, in a market with average characteristics, which translates into 12.5 and 3.3 percentage point decreases. ${ }^{26}$ Since each provider generally chooses different levels of capacity, a rival's presence should have a nonuniform effect across markets, and the competition should be more intense when the rival chooses a higher capacity level. A model that focuses on discrete market entry decisions would have overlooked these heterogeneous competitive interactions.

The negative impact of competitors' capacities on a provider's profits may come from several distinct channels. First, additional competitors affect a provider's profits by splitting the existing customers from that provider. By diverting patients away from incumbents, the presence of an additional competitor may lower the utilization of the facilities, increase the variable cost per unit in capacity, and lead to a lower margin per unit in capacity. Second, competition can also lead to a lower price for privately insured patients (Cutler et al., 2012), and therefore lowers the average per patient margin. Third, providers also compete for a limited supply of trained personnel (e.g., nurses and technicians) who operate dialysis stations to generate revenues. Fourth, for each provider, competitors with a higher capacity are likely to offer more flexible schedules, holding other things fixed (e.g., utilization rates), and thus have greater negative impact in terms of splitting customers. With more detailed patientand facility-level data, it is possible to conduct a full structural analysis of patients' choices on the demand side, and thus distinguish the impacts of competitors' capacity through these distinct channels.

Most of the coefficients in table 4 are significant, with expected signs. Market size, extent of the diabetic population, and supply of local nephrologists and registered nurses are positively associated with the capacity of all providers, while entry barriers such as CON regulation are negatively associated with capacity choices. Some market conditions may be important for one firm but not for others. This implies that dialysis firms target different demographics, and it is important to account for firm heterogeneity. Distance to headquarters does not seem to be very important to the capacity

\footnotetext{
26 The percentage points are computed based on the mean entry probability of 0.24 and 0.30 for DaVita and nonchain, respectively. The 0.03 points decrease in probability is equivalent to $0.03 / 0.24=12.5 \%$ in percentage points.
}

TABle 5.-EQuilibrium ResPonse to MARKet VARIABLeS

\begin{tabular}{|c|c|c|c|c|c|c|}
\hline & \multicolumn{3}{|c|}{ Capacity } & \multicolumn{3}{|c|}{ Number of HEP Markets } \\
\hline & FMC & DaVita & Nonchain & FMC & DaVita & Nonchain \\
\hline base case & 8,003 & 5,586 & 6,700 & 330 & 115 & 214 \\
\hline $\begin{array}{l}\text { Population } \\
\quad \text { increases } 10 \%\end{array}$ & 8,560 & 6,097 & 7,238 & 368 & 137 & 246 \\
\hline $\begin{array}{l}\text { Nephrologists } \\
\text { increase } 10 \%\end{array}$ & 8,066 & 5,784 & 6,766 & 337 & 126 & 216 \\
\hline $\begin{array}{l}\text { Prevalence rate of } \\
\text { diabetes } \\
\text { increases } 10 \%\end{array}$ & 8,996 & 5,593 & 6,565 & 395 & 107 & 202 \\
\hline $\begin{array}{c}\mathrm{CON} \text { regulation }=0 \\
\text { for all markets }\end{array}$ & 8,336 & 5,593 & 6,795 & 354 & 114 & 216 \\
\hline
\end{tabular}

Number of observations: 1,320. Capacity is derived from equation (8) after adjusting market variables. The "Capacity" column reports the sum of expected capacity choice for each provider across markets. The number of HEP (high-entry probability) markets for a provider is defined as the number of markets in which that provider's entry probability is estimated to be greater than 0.5 . It is reported in the "Number of HEP Markets" column.

choice of the two leading chain providers. ${ }^{27}$ But nonchain providers are more likely to offer positive capacity when the market is farther away from either chain's headquarters.

To better understand the magnitude of these estimates, table 5 reports the equilibrium response in capacity and the number of markets with high entry probabilities (i.e., greater than $50 \%$ ) based on the GMM estimates when some marketlevel variables (i.e., population, the number of nephrologists, and the diabetes prevalence rate) change. ${ }^{28}$ To derive the effect on capacity, we resolve the equilibrium based on equation (8) after adjusting these market-level variables upward by $10 \%$. The equilibrium entry probability of a provider on a market characterized by $x$ is computed as

$$
\operatorname{Pr}\left(K_{i}^{*}>0 \mid x\right)=\Phi\left[\left(x \beta_{i}+\sum_{j \neq i} \gamma_{i, j} \mathbb{E}\left[K_{j}^{*} \mid x\right]-b_{i}\right) / \sigma_{i}\right]
$$

and $E\left(K^{*} \mid X\right)$ is the new equilibrium capacity after the adjustment of market variables. We also calculate a measure of provider-specific market penetration under these hypothetical adjustments. Such a measure is defined as the proportion of markets in the sample where a provider would enter with probability greater than $50 \%$ under the adjustment considered.

The effect of population is quite substantial. When the population increases by $10 \%$, the total capacity unambiguously increases by $7 \%, 9 \%$, and $8 \%$ for FMC, DaVita, and nonchain, while the market penetration (as measured by the number of markets with high entry probabilities for a provider) increase by $12 \%, 19 \%$, and $15 \%$, respectively. There are mild increases in capacity and market penetration measures when the number of nephrologists increases by $10 \%$. The effect on nonchain providers is the smallest. This is probably not too

\footnotetext{
${ }^{27}$ Distance to FMC's headquarter is marginally significant for DaVita. All other distance variables are insignificant for the two chain providers.

28 The two-stage and maximum likelihood estimates generate very similar outcomes.
} 
surprising since many nonchain dialysis facilities are owned and operated by local nephrologist groups. By contrast, chain facilities benefit more from a larger nephrologist stock since such stocks make it easier to locate medical directors with an established referral base. The increase in the prevalence rate of diabetes has heterogeneous effects on different providers. While FMC increases the capacity substantially, nonchain providers reduce the capacity, while the capacity of DaVita remains almost constant. This seems to suggest that FMC exerts an intense competitive pressure on its competitors. In general, a larger diabetic patient base in a market increases the profitability of the dialysis business as a whole. However, for DaVita or nonchain providers, this positive effect on profits is neutralized or reversed by an offsetting competition effect due to the expansion of FMC in the new market equilibrium. Such a competition effect is evident from our estimates for the negative strategic effects of capacity, which imply downward-sloping reaction curves for all providers. A similar intuition could explain why both DaVita and nonchain respond mildly to the repeal of $\mathrm{CON}$ regulation by increasing its capacity, while FMC increases its capacity substantially.

As robustness checks, we estimate the model using a larger sample with 3,129 markets (HSAs) with no population restrictions and the two-stage estimator. We report the results in panel A of table A1 in the appendix. This includes $92 \%$ of all HSAs in the continental United States. In addition, we experimented with alternative sample selection criteria by focusing on markets with total capacity below 80 or 40 (instead of 60 as in panel A). The results in panels B and $\mathrm{C}$ of table $\mathrm{A} 1$ remain qualitatively similar to those in table 4.

It is worth noting that the results from panel A in table A1 also help to justify indirectly the assumption that providers' private information is independent. To see this, suppose providers' private information is correlated with some market conditions that are not reported in the data but are commonly known to all providers (e.g., a limited supply of experienced nurses or managers). If such omitted conditions vary across markets and are known to be correlated with other market characteristics reported in data, then we should expect to obtain very different estimates of coefficients in the profit function using samples that differ in these reported market characteristics. In our context, it is plausible that unobserved conditions (such as the limited supply of qualified facility managers and staffs) are correlated with the population density of the markets. Table A1 (panel A) reports estimates of strategic coefficients using the two-stage estimator in an enlarged sample with 3,129 markets, which include those with substantially different population densities. These estimates are very similar to those based on a smaller sample in table 4 (panel A). This offers some evidence that the independent private information assumption works reasonably well in our setting.

\begin{tabular}{|c|c|c|c|c|c|c|}
\hline & \multicolumn{3}{|c|}{ Capacity } & \multicolumn{3}{|c|}{ Entry Probability } \\
\hline & FMC & DaVita & Nonchain & FMC & DaVita & Nonchain \\
\hline \multicolumn{7}{|l|}{ Observed } \\
\hline Mean & 6.40 & 4.40 & 5.32 & 0.31 & 0.23 & 0.28 \\
\hline SD & 11.4 & 9.23 & 10.0 & 0.46 & 0.42 & 0.45 \\
\hline \multicolumn{7}{|c|}{ Two-stage } \\
\hline Mean & 6.24 & 4.15 & 5.13 & 0.34 & 0.24 & 0.30 \\
\hline SD & 5.56 & 4.18 & 4.62 & 0.22 & 0.18 & 0.19 \\
\hline \multicolumn{7}{|c|}{ Maximum likelihood } \\
\hline Mean & 6.23 & 4.16 & 4.81 & 0.34 & 0.24 & 0.27 \\
\hline SD & 7.68 & 5.82 & 5.56 & 0.22 & 0.18 & 0.20 \\
\hline \multicolumn{7}{|l|}{ GMM } \\
\hline Mean & 6.06 & 4.23 & 5.08 & 0.35 & 0.24 & 0.30 \\
\hline SD & 5.33 & 4.46 & 4.16 & 0.21 & 0.18 & 0.18 \\
\hline
\end{tabular}

Number of observations: 1,320. Mean reports the sample average. SD reports the standard deviation. The Observed panel reports the statistics from data, and the Two-Stage, ML, and GM panels report the model prediction from three estimators, respectively.

\section{B. Model Fit}

We compute the fitted (implied) values of capacity choices and entry probabilities using three sets of estimates $\hat{\theta}^{T S}, \hat{\theta}^{F L}$, and $\hat{\theta}^{G M}$, respectively, and compare them with observed outcomes in the data. Overall, the three estimators produce very similar results. Such a similarity is not surprising ex post, because these estimators essentially use the same set of information. ${ }^{29}$ These predictions also suggest that our model fits the data quite well.

First, we compute the expected capacity choice $\mathbb{E}\left(K^{*} \mid X\right)$ implied by model estimates. These expectations are solutions to fixed-point equation (8) based on the parameter estimates. We find the predicted expected capacity from all three estimators is close to the average capacity in data. For example, the average capacities in data are 6.40, 4.40, and 5.32 for each provider, while our GMM estimates predict 6.06, 4.23, and 5.08 respectively. Results obtained from the two-stage and maximum likelihood estimates are similar. This suggests that our model estimates explain capacity choices quite well on average.

Second, we compute the implied entry probabilities for each provider and compare them with entry probabilities observed in data. The predicted entry probabilities are calculated using equation (11) where $\mathbb{E}\left[K_{i}^{*} \mid x\right]$ solves the fixedpoint equation in equation (8). The rows in table 6 report the means and the standard deviations of such probabilities for each provider. The predicted entry probabilities are close to the entry proportions reported in data. For example, the empirical entry probability for DaVita is 0.23 in data, while our model predicts an average entry probability of 0.24 . We also impute binary entry decisions based on whether the

\footnotetext{
${ }^{29}$ Even in the ML estimator, the MPEC algorithm implicitly uses data to guide through multiple equilibria by always picking the vector of parameters and strategies that maximizes the likelihood. GMM estimator is computationally different from ML since one set of moments consists of the first-order conditions (in analytical form) for maximizing the likelihood. Depending on the shape of the likelihood function around the true value of parameters, GMM estimates may be less susceptible to "optimization errors" in the minimization routine.
} 
implied entry probability of a market is greater than $50 \%$. Based on such imputations and GMM estimates, we find our model correctly predicts the binary entry decisions in $70 \%, 78 \%$, and $73 \%$ of the markets for FMC, DaVita, and nonchain, respectively. Similarly, the proportions of correct predictions are $70 \%, 78 \%$, and $65 \%$ based on the two-stage estimates and $65 \%, 77 \%$ and $74 \%$ based on the maximum likelihood estimates. Overall, our model does well in dealing with the censoring in capacity data.

Finally, we extrapolate from our sample and use our estimates to predict the capacity choices in 1,809 medium to small HSAs that are not currently included in the sample used in table $4 .{ }^{30}$ Our model also does a good job in the out-of-sample prediction. Appendix table A2 presents the comparison between the implied and the observed outcomes in the out-of-sample markets.

\section{Counterfactual Analyses}

Our counterfactual experiment is motivated by policy debates on how to curb the rapid growth of dialysis expenditure. In 2011, Medicare started to implement a new bundled dialysis payment system that incorporates the formerly separate billable items into a new bundled flat rate. ${ }^{31}$ This effectively lowers the dialysis providers' margin per treatment. To capture this policy effect, let $\lambda$ be a multiplication factor for variable profits and $\Delta$ for the costs.

The counterfactual payoffs under the alternative profit and cost factors are

$$
\begin{aligned}
\Pi_{i}= & \lambda K_{i}\left(x \beta_{i}+\sum_{j \neq i} \gamma_{i, j} \mathbb{E}_{\varepsilon_{j}}\left[K_{j}\left(x, \varepsilon_{j}\right) \mid x\right]-\varepsilon_{i}\right) \\
& -\Delta\left(a_{i} K_{i}^{2}+b_{i} K_{i}\right) .
\end{aligned}
$$

As shown in section III, under the scale normalization $a_{i}=$ $1 / 2$, the counterfactual equilibrium choices of capacity for provider $i$ (denoted by $\tilde{K}_{i}$ ) must satisfy

$$
\begin{aligned}
\tilde{K}_{i}\left(x, \varepsilon_{i}\right)= & \max \left\{0, \frac{\lambda}{\Delta}\right. \\
& \left.\left(x \beta_{i}+\sum_{j \neq i} \gamma_{i, j} \mathbb{E}_{\varepsilon_{j}}\left[\tilde{K}_{j}\left(x, \varepsilon_{j}\right) \mid x\right]-\varepsilon_{i}\right)-b_{i}\right\} .
\end{aligned}
$$

\footnotetext{
30 These markets are used to perform robustness analysis in panel A of appendix A1. There are 3,129-1,320 =1,809 markets, where, 1,320 is the number of markets in the sample used in table 4.

31 The new system also incorporates some pay-for-performance incentives. Penalties will be imposed on providers whose dialysis quality measures (patients' hemoglobin and urea levels) did not meet standards. The maximum payment reduction is up to $2 \%$. We did not explicitly investigate the pay-for-performance incentive in our analysis for two reasons. First, the incentive is relatively small. Second, several papers (Grieco \& McDevitt, 2014; Cutler et al., 2012) find that dialysis quality is not sensitive to competition.
}

For all $i$, let $\tilde{\varphi}_{i}=\mathbb{E}_{\varepsilon_{i}}\left(\tilde{K}_{i}\left(x, \varepsilon_{i}\right) \mid x\right)$ be the expected counterfactual capacity defined by the following equilibrium condition:

$$
\begin{gathered}
\tilde{\varphi}_{i}=\Phi\left(\frac{x \beta_{i}+\sum_{j \neq i} \gamma_{i, j} \tilde{\varphi}_{j}-b_{i} \frac{\Delta}{\lambda}}{\sigma_{i}}\right) * \frac{\lambda}{\Delta} \\
*\left\{x \beta_{i}+\sum_{j \neq i} \gamma_{i, j} \tilde{\varphi}_{j}-b_{i} \frac{\Delta}{\lambda}\right. \\
\left.+\sigma_{i} \frac{x\left(\frac{x \beta_{i}+\sum_{j \neq i} \gamma_{i, j} \tilde{\varphi}_{j}-b_{i} \frac{\Delta}{\lambda}}{\sigma_{i}}\right)}{\Phi\left(\frac{x \beta_{i}+\sum_{j \neq i} \gamma_{i, j} \tilde{\varphi}_{j}-b_{i} \frac{\Delta}{\lambda}}{\sigma_{i}}\right)}\right\} .
\end{gathered}
$$

The predictions reported in table 6 are for the status quo in data (when $\lambda=1, \Delta=1$ ). If the costs per unit in capacity increase by $20 \%$ and variable profits per unit incapacity decrease by $10 \%$, the counterfactual capacity distribution is obtained by adjusting $\frac{\lambda}{\Delta}=\frac{0.9}{12}=0.75$ in equation (14). A similar counterfactual was applied by Schaumans and Verboven (2008) to investigate the market for health care professionals. According to MedPAC, the base composite rate under the old system is about $\$ 142$ per patient in 2012 (after excluding the $\$ 20$ drug add-on payment). Since the separate billable drugs account for approximately $40 \%$ of total Medicare payment for dialysis, this leads to an estimated average of $\$ 257$ per treatment payment. Given the new composite payment base rate of $\$ 235$ in the same year, there is approximately a $5 \%$ reduction in the per patient payment. Due to the lack of detailed data on patient-level payments and costs, we cannot precisely measure the reduction on the per patient margin as a result of the reduction in the payment rate. Instead, we qualitatively investigate the new policy by lowering $\frac{\lambda}{\Delta}$ to different levels. ${ }^{32}$ In table 7, we report the predicted equilibrium outcome when $\frac{\lambda}{\Delta}$ is reduced by $2 \%, 5 \%$, and $8 \%$. Possibilities also remain that the margin goes up for facilities that do not rely too much on separate billable drugs. We also simulate the outcome when $\frac{\lambda}{\Delta}$ increases by $2 \%$. Finally, we simulate the heterogeneous response to the policy reform by adjusting $\frac{\lambda}{\Delta}=98 \%$ for DaVita while holding the ratio $\frac{\lambda}{\Delta}$ constant for other providers. This is motivated by the established fact that DaVita relies heavily on drug revenues.

Table 7 presents predicted results for different counterfactual scenarios based on our GMM estimates. ${ }^{33}$ The first

\footnotetext{
32 While it is clear that $\lambda / \Delta$ is closely related to the Medicare payment rate, the exact mapping between the two $\lambda / \Delta$ is not transparent due to our model specifications and, in particular, the lack of facility-level data. If facility-level data are available, one could regress the imputed provider profits implied by our model against the observed facility profits, come up with a factor that relates the model implied margin to the observed margin, and use this factor to infer the magnitude of the change in $\lambda / \Delta$ as a result from the new policy. Our counterfactual analysis qualitatively investigates the effect of the new policy by lowering $\lambda / \Delta$ to various levels.

${ }^{33}$ Both the two-stage and maximum likelihood estimates give predictions that are very similar to the GMM estimates. To economize on space, we report in this paper only the results obtained from the GMM estimates.
} 
TABle 7.-COUnTerfactual CAPACITy Distribution

\begin{tabular}{|c|c|c|c|c|c|c|c|c|c|}
\hline & \multicolumn{3}{|c|}{ E(Capacity) } & \multicolumn{3}{|c|}{$\operatorname{Pr}($ Entry) } & \multicolumn{3}{|c|}{ E(Capacity | Entry) } \\
\hline & FMC & DaVita & Nonchain & FMC & DaVita & Nonchain & FMC & DaVita & Nonchain \\
\hline$\lambda / \Delta$ & $100 \%$ & $100 \%$ & $100 \%$ & $100 \%$ & $100 \%$ & $100 \%$ & $100 \%$ & $100 \%$ & $100 \%$ \\
\hline 25 th & 2.10 & 0.16 & 1.91 & 0.18 & 0.08 & 0.16 & 11.6 & 11.4 & 12.0 \\
\hline 50 th & 4.76 & 3.06 & 4.19 & 0.34 & 0.21 & 0.29 & 14.1 & 14.3 & 14.3 \\
\hline 75 th & 8.50 & 6.11 & 7.18 & 0.50 & 0.36 & 0.43 & 17.0 & 17.1 & 16.7 \\
\hline Mean & 6.06 & 4.23 & 5.08 & 0.35 & 0.24 & 0.30 & 14.6 & 14.5 & 14.5 \\
\hline SD & 5.33 & 4.46 & 4.16 & 0.21 & 0.18 & 0.18 & 4.45 & 4.44 & 3.81 \\
\hline Total & 8,237 & 5,473 & 6,774 & 321 & 131 & 210 & 19,286 & 19,084 & 19,099 \\
\hline$\lambda / \Delta$ & $98 \%$ & $98 \%$ & $98 \%$ & $98 \%$ & $98 \%$ & $98 \%$ & $98 \%$ & $98 \%$ & $98 \%$ \\
\hline 25 th & 1.36 & 0.57 & 1.03 & 0.13 & 0.05 & 0.10 & 10.4 & 10.4 & 10.5 \\
\hline 50th & 3.54 & 2.45 & 2.62 & 0.28 & 0.18 & 0.21 & 12.8 & 13.4 & 12.6 \\
\hline 75 th & 6.70 & 5.91 & 4.81 & 0.43 & 0.36 & 0.33 & 15.4 & 16.6 & 14.6 \\
\hline Mean & 4.60 & 4.00 & 3.34 & 0.29 & 0.23 & 0.23 & 13.1 & 13.8 & 12.4 \\
\hline SD & 4.20 & 4.59 & 2.99 & 0.20 & 0.19 & 0.16 & 3.80 & 4.67 & 3.17 \\
\hline Total & 6,069 & 5,280 & 4,408 & 218 & 141 & 72 & 17,335 & 18,237 & 16,679 \\
\hline$\lambda / \Delta$ & $95 \%$ & $95 \%$ & $95 \%$ & $95 \%$ & $95 \%$ & $95 \%$ & $95 \%$ & $95 \%$ & $95 \%$ \\
\hline 25 th & 0.60 & 0.20 & 0.30 & 0.06 & 0.22 & 0.04 & 8.81 & 8.84 & 8.62 \\
\hline 50 th & 1.90 & 1.40 & 1.10 & 0.18 & 0.12 & 0.10 & 10.9 & 11.8 & 10.3 \\
\hline 75 th & 4.30 & 4.50 & 2.40 & 0.32 & 0.30 & 0.20 & 13.2 & 15.1 & 12.0 \\
\hline Mean & 2.8 & 3.1 & 1.6 & 0.21 & 0.18 & 0.13 & 11.1 & 12.3 & 10.3 \\
\hline SD & 2.80 & 4.20 & 1.70 & 0.17 & 0.19 & 0.11 & 9.59 & 4.58 & 2.47 \\
\hline Total & 3,727 & 4,135 & 2,116 & 90 & 115 & 8 & 14,666 & 16,288 & 13,642 \\
\hline$\lambda / \Delta$ & $92 \%$ & $92 \%$ & $92 \%$ & $92 \%$ & $92 \%$ & $92 \%$ & $92 \%$ & $92 \%$ & $92 \%$ \\
\hline 25 th & 0.18 & 0.04 & 0.07 & 0.02 & 0.01 & 0.01 & 7.36 & 7.38 & 7.08 \\
\hline 50th & 0.83 & 0.55 & 0.33 & 0.09 & 0.06 & 0.04 & 9.10 & 9.84 & 8.41 \\
\hline 75 th & 2.39 & 2.57 & 0.95 & 0.21 & 0.20 & 0.10 & 11.1 & 12.9 & 9.82 \\
\hline Mean & 1.56 & 2.01 & 0.65 & 0.13 & 0.13 & 0.06 & 9.31 & 10.5 & 8.45 \\
\hline SD & 1.84 & 3.16 & 0.83 & 0.13 & 0.16 & 0.07 & 2.57 & 4.06 & 1.95 \\
\hline Total & 2,060 & 2,650 & 859 & 10 & 61 & 0 & 12,299 & 13,885 & 11,156 \\
\hline$\lambda / \Delta$ & $102 \%$ & $102 \%$ & $102 \%$ & $102 \%$ & $102 \%$ & $102 \%$ & $102 \%$ & $102 \%$ & $102 \%$ \\
\hline 25 th & 2.99 & 1.25 & 3.17 & 0.24 & 0.10 & 0.23 & 12.7 & 12.2 & 13.5 \\
\hline 50th & 6.09 & 3.32 & 6.30 & 0.40 & 0.22 & 0.39 & 15.4 & 14.8 & 16.3 \\
\hline 75 th & 10.5 & 5.76 & 10.3 & 0.56 & 0.34 & 0.53 & 18.6 & 17.0 & 19.2 \\
\hline Mean & 7.69 & 4.14 & 7.37 & 0.41 & 0.34 & 0.38 & 16.2 & 14.8 & 16.6 \\
\hline $\mathrm{SD}$ & 6.55 & 4.07 & 5.60 & 0.22 & 0.16 & 0.21 & 5.21 & 4.03 & 4.63 \\
\hline Total & 10,148 & 5,470 & 9,722 & 446 & 86 & 406 & 21,330 & 19,521 & 21,917 \\
\hline$\lambda / \Delta$ & $100 \%$ & $98 \%$ & $100 \%$ & $100 \%$ & $98 \%$ & $100 \%$ & $100 \%$ & $98 \%$ & $100 \%$ \\
\hline 25 th & 2.20 & 0.40 & 1.97 & 0.19 & 0.04 & 0.16 & 11.7 & 9.92 & 12.0 \\
\hline 50th & 5.24 & 1.43 & 4.38 & 0.36 & 0.12 & 0.30 & 14.5 & 12.1 & 14.5 \\
\hline 75 th & 9.91 & 2.82 & 7.61 & 0.55 & 0.20 & 0.45 & 18.0 & 13.8 & 17.1 \\
\hline Mean & 6.89 & 1.84 & 5.41 & 0.38 & 0.13 & 0.31 & 15.2 & 11.8 & 14.7 \\
\hline SD & 6.24 & 1.77 & 4.53 & 0.23 & 0.10 & 0.19 & 5.09 & 2.76 & 4.05 \\
\hline Total & 9,095 & 2,427 & 7,138 & 403 & 6 & 251 & 20,100 & 15,610 & 19,440 \\
\hline
\end{tabular}

Number of observations: 1,320 . Under status quo, $\lambda / \Delta=100 \%$ for all providers. The 25 th, 50 th, and 75 th rows report the corresponding quartile of distributions. The mean capacity row reports the average expected capacity across markets. The total capacity row reports the sum of expected capacity across markets. The mean entry row reports the average entry probability across markets. The total entry probability row reports the total number of markets with high entry probability (i.e., entry probability greater than 0.5 ).

three columns for $\mathrm{E}$ (Capacity) give summary statistics (the mean, the quartiles, and the standard deviation) of the empirical distribution of expected equilibrium capacities predicted for each market. The two other main columns, E(Entry) and $\mathrm{E}$ (Capacity | Entry), present summary statistics from the predicted distribution of counterfactual entry probabilities, as well as the expected capacity choice conditional on entry for each provider.

For each provider, the quartiles and the mean under the new policy are almost always decreasing in the ratio experimented with. For example, for FMC, the median of the expected equilibrium choices of capacity predicted for 1,320 markets in the data is 4.76 for the status quo $(\lambda / \Delta=100 \%)$ but decreases to 3.54 or 0.83 when $\lambda / \Delta=98 \%$ or $92 \%$. The same pattern shows up for the other providers uniformly across all ratios experimented with. This seems to suggest that the counterfactual distribution of expected capacity choices is stochastically decreasing in the size of the reduction in the ratio $\lambda / \Delta$. This conforms with the intuition that providers reduce their capacity choices in response to reductions in the margin.

The magnitudes of changes in response to the adjusted margins, however, are heterogeneous across providers. The size of capacity changes at DaVita and FMC are less drastic than that for nonchain providers. For example, for nonchain providers, the median of the expected capacity increases from 4.19 to 6.30 when the ratio $\lambda / \Delta$ increases from the status quo to $102 \%$ and reduces to 0.33 when the ratio drops to $92 \%$. In comparison, for DaVita, the median of the expected capacity increases from 3.06 to 3.32 when the ratio $\lambda / \Delta$ increases from the status quo to $102 \%$ and reduces to 0.55 when the markup ratio drops to $92 \%$. This seems to suggest the local 
independent and nonchain providers are affected the most by a policy reform that reduces the margin for all providers uniformly by the same proportion.

Another finding from our estimates is that the capacity adjustment of DaVita tends to be negatively correlated with that of FMC. In fact, DaVita increases its capacity stock in 108 markets when $\frac{\lambda}{\Delta}=95 \%$. This pattern could be ascribed to the downward-sloping reaction curve that results from the strategic interactions in capacity choices. When a competitor reduces its capacity, the downward-sloping reaction curve incentivizes a provider to increase its capacity. It also helps to mitigate the negative impact on providers' capacity choices and market presence when there are negative profit shocks.

The entry probabilities respond in a similar manner when the margin for all providers decreases by the same proportion. Nonchain providers respond more strongly than FMC and DaVita. Though the average entry probability for DaVita decreases unambiguously with a lower profit-to-cost ratio $\frac{\lambda}{\Delta}$, the number of markets with high entry probability increases substantially for DaVita when $\frac{\lambda}{\Delta}=98 \%$. Nevertheless, when the ratio is reduced further to $\frac{\lambda}{\Delta}=95 \%$, the number of such markets with high entry probabilities is remarkably lower than that under the status quo for DaVita. ${ }^{34}$

Since the policy reform can imply a positive profit shock for providers that are less reliant on drug revenues, we also investigate the outcome when $\frac{\lambda}{\Delta}=102 \%$ for all providers. FMC responds with more substantial increase in its capacity than nonchain and DaVita. All in all, when the providers share the same market-wise profit shocks (such as a reduction in the margin induced by the new payment policy), DaVita is expected to be more resistant to negative profit shocks and less responsive to positive profit shocks. This may arise from the asymmetries in providers' profit and cost structures, as well as the difference in their strategic responses to their rivals' capacity choices.

We then study the predictions under a provider-specific policy reform where there is a negative profit shock for DaVita and no shocks for FMC and the nonchain providers. Specifically, we set $\frac{\lambda}{\Delta}=0.98$ for DaVita and $\frac{\lambda}{\Delta}=1$ for FMC and nonchain providers. ${ }^{35}$ Our model predicts that DaVita reduces capacity stock substantially, while both rivals slightly increase their capacities. The negative profit shock for DaVita is magnified by the downward-sloping reaction curve. When

\footnotetext{
${ }^{34}$ The counterfactual analysis in table 7 also suggests that a small decrease in margin could induce significant reduction in capacity choices and entry probabilities. This is probably because even a small percentage reduction in markup on each dialysis station could sum up to a significant amount given that an average facility owns eighteen to twenty stations. Another possible explanation of the large estimated effect of the policy change is that in most markets in our data, the markups are quite high (the differences between variable profits and fixed costs are reasonably large) so that a small discrepancy in the change of $\lambda$ and $\Delta$ would affect the profitability of providers nontrivially. This effect is also compounded through the strategic interactions between providers.

35 As we explained previously, this is motivated by the observation that DaVita relies heavily on drug revenue. In 2007, the New York Times (Pollack, 2007) reported that $40 \%$ of DaVita's revenue comes from dialysis-related drugs. In comparison, 25\% of FMC's revenue depends on drugs.
}

the margin decreases, a direct response for DaVita is to reduce capacity. This motivates both FMC and nonchain to increase their capacity through the reaction curve, which serves as an additional incentive for DaVita to reduce capacity in the new equilibrium. Overall, our model suggests that with the downward-sloping reaction curve, even a seemingly small asymmetric profit shock could induce significant changes in dialysis providers' capacity choices.

We also report outcomes of counterfactual experiments for markets with a different population and prevalence rates of diabetes. Table 8 presents the mean and the standard deviation of counterfactual expected capacity choices and entry probabilities in heterogeneous groups of markets with different population or prevalence rates of diabetes. Our goal is to learn about the distribution of counterfactual distributions of expected capacity choices and entry probabilities in different markets if Medicare were to adopt discriminatory reimbursement policies based on these observed market attributes.

We continue to find heterogeneity across FMC, DaVita, and nonchain providers in their responses to margin reductions conditional on market characteristics. In addition, there is strong evidence that responses differ substantially across markets with different population or diabetes prevalence rates. For instance, if the profit margins measured by $\lambda / \Delta$ are reduced by $2 \%$ for markets with population above the median, then the average of expected capacity choices in equilibrium will be reduced to 6.92, 6.76, and 5.32 for FMC, DaVita, and nonchain providers, respectively. In this case, the average expected capacity choices will be reduced to $2.32,1.29$, and 1.39 , respectively, in markets with a population below the median.

It is also worth noting that other things being equal, the counterfactual distributions of expected choices of capacity and entry probabilities in markets with higher population or diabetes prevalence rates stochastically dominate those in markets with lower population or prevalence rates. This pattern could be explained by the fact that profits from markets with larger consumer bases respond more dramatically to changes in profit margins.

\section{Conclusion}

Dialysis providers in the United States usually choose the scale of their operation (as measured by the number of dialysis stations) when they start to serve a market. Such capacity choices rarely change after the initial entry and vary substantially across providers and markets. To capture these empirical regularities, we propose a structural model of Bayesian games with continuous actions and use it to estimate providers' payoff structure and, in particular, the strategic interaction between providers' capacity choices. We estimate the model using several estimators, including a GMM estimator that fully exploits the structural relationship. We use the estimates to investigate counterfactual policy interventions that change providers' profit margins per unit in capacity. 
Table 8.-Counterfactual Capacity Distribution by Market Types

\begin{tabular}{|c|c|c|c|c|c|c|c|c|c|c|}
\hline \multirow[b]{2}{*}{ Market } & & \multicolumn{3}{|c|}{ E(Capacity) } & \multicolumn{3}{|c|}{$\operatorname{Pr}$ (Entry) } & \multicolumn{3}{|c|}{ E(Capacity | Entry) } \\
\hline & & FMC & DaVita & Nonchain & FMC & DaVita & Nonchain & FMC & DaVita & Nonchain \\
\hline$\lambda / \Delta$ & & $100 \%$ & $100 \%$ & $100 \%$ & $100 \%$ & $100 \%$ & $100 \%$ & $100 \%$ & $100 \%$ & $100 \%$ \\
\hline \multirow[t]{2}{*}{ pop below $50 \%$} & Mean & 3.30 & 1.62 & 2.36 & 0.23 & 0.11 & 0.17 & 12.2 & 11.6 & 11.9 \\
\hline & $\mathrm{SD}$ & 3.42 & 1.81 & 1.97 & 0.17 & 0.11 & 0.12 & 3.30 & 2.90 & 2.52 \\
\hline \multirow[t]{2}{*}{ pop above $50 \%$} & Mean & 8.87 & 6.89 & 7.84 & 0.48 & 0.36 & 0.43 & 17.1 & 17.4 & 17.1 \\
\hline & $\mathrm{SD}$ & 5.45 & 4.78 & 3.97 & 0.18 & 0.16 & 0.14 & 4.06 & 3.76 & 3.05 \\
\hline \multirow[t]{2}{*}{$\mathrm{db}$ rate below $50 \%$} & Mean & 4.10 & 3.69 & 5.07 & 0.27 & 0.21 & 0.30 & 13.0 & 13.7 & 14.4 \\
\hline & $\mathrm{SD}$ & 3.52 & 4.37 & 4.43 & 0.18 & 0.19 & 0.20 & 3.35 & 4.56 & 4.04 \\
\hline \multirow[t]{2}{*}{$\mathrm{db}$ rate above $50 \%$} & Mean & 7.99 & 4.76 & 5.08 & 0.43 & 0.27 & 0.31 & 16.2 & 15.2 & 14.5 \\
\hline & $\mathrm{SD}$ & 6.05 & 4.50 & 3.89 & 0.22 & 0.17 & 0.17 & 4.78 & 4.19 & 3.56 \\
\hline \multicolumn{2}{|l|}{$\lambda / \Delta$} & $98 \%$ & $98 \%$ & $98 \%$ & $98 \%$ & $98 \%$ & $98 \%$ & $98 \%$ & $98 \%$ & $98 \%$ \\
\hline \multirow[t]{2}{*}{ pop below $50 \%$} & Mean & 2.32 & 1.29 & 1.39 & 0.17 & 0.09 & 0.12 & 10.9 & 10.7 & 10.5 \\
\hline & $\mathrm{SD}$ & 2.62 & 1.69 & 1.31 & 0.15 & 0.10 & 0.09 & 2.88 & 2.90 & 2.15 \\
\hline \multirow[t]{2}{*}{ pop above $50 \%$} & Mean & 6.92 & 6.76 & 5.32 & 0.41 & 0.36 & 0.34 & 15.4 & 17.0 & 14.8 \\
\hline & $\mathrm{SD}$ & 4.23 & 4.95 & 2.91 & 0.16 & 0.17 & 0.13 & 3.24 & 3.94 & 2.43 \\
\hline \multirow[t]{2}{*}{$\mathrm{db}$ rate below $50 \%$} & Mean & 3.11 & 3.30 & 3.30 & 0.22 & 0.19 & 0.22 & 11.8 & 12.9 & 12.5 \\
\hline & $\mathrm{SD}$ & 2.86 & 4.24 & 3.16 & 0.16 & 0.19 & 0.17 & 3.05 & 4.61 & 3.35 \\
\hline \multirow[t]{2}{*}{$\mathrm{db}$ rate above $50 \%$} & Mean & 6.06 & 4.69 & 3.38 & 0.36 & 0.26 & 0.23 & 14.5 & 14.7 & 12.8 \\
\hline & $\mathrm{SD}$ & 4.75 & 4.81 & 2.81 & 0.20 & 0.19 & 0.15 & 3.99 & 4.55 & 2.99 \\
\hline \multicolumn{2}{|l|}{$\lambda / \Delta$} & $95 \%$ & $95 \%$ & $95 \%$ & $95 \%$ & $95 \%$ & $95 \%$ & $95 \%$ & $95 \%$ & $95 \%$ \\
\hline \multirow[t]{2}{*}{ pop below $50 \%$} & Mean & 1.22 & 0.77 & 0.54 & 0.11 & 0.06 & 0.05 & 9.17 & 9.26 & 8.60 \\
\hline & $\mathrm{SD}$ & 1.63 & 1.33 & 0.62 & 0.11 & 0.09 & 0.05 & 2.33 & 2.69 & 1.69 \\
\hline \multirow[t]{2}{*}{ pop above $50 \%$} & Mean & 4.46 & 5.54 & 2.69 & 0.31 & 0.31 & 0.21 & 13.1 & 15.5 & 12.1 \\
\hline & $\mathrm{SD}$ & 2.88 & 4.66 & 1.72 & 0.15 & 0.18 & 0.10 & 2.46 & 3.93 & 1.80 \\
\hline $\mathrm{db}$ rate below $50 \%$ & Mean & 1.88 & 2.39 & 1.55 & 0.15 & 0.15 & 0.12 & 10.1 & 11.3 & 10.2 \\
\hline & $\mathrm{SD}$ & 2.00 & 3.53 & 1.75 & 0.13 & 0.17 & 0.12 & 2.64 & 4.31 & 2.58 \\
\hline $\mathrm{db}$ rate above $50 \%$ & Mean & 3.75 & 3.86 & 1.65 & 0.26 & 0.22 & 0.13 & 12.1 & 13.3 & 10.5 \\
\hline & $\mathrm{SD}$ & 3.21 & 4.59 & 1.60 & 0.18 & 0.20 & 0.11 & 3.17 & 4.63 & 2.34 \\
\hline$\lambda / \Delta$ & & $92 \%$ & $92 \%$ & $92 \%$ & $92 \%$ & $92 \%$ & $92 \%$ & $92 \%$ & $92 \%$ & $92 \%$ \\
\hline pop below $50 \%$ & Mean & 0.54 & 0.36 & 0.16 & 0.05 & 0.03 & 0.02 & 7.65 & 7.80 & 7.07 \\
\hline & $\mathrm{SD}$ & 0.90 & 0.85 & 0.23 & 0.07 & 0.06 & 0.02 & 1.85 & 2.28 & 1.31 \\
\hline pop above $50 \%$ & Mean & 2.60 & 3.68 & 1.15 & 0.21 & 0.23 & 0.11 & 11.0 & 13.3 & 9.86 \\
\hline & $\mathrm{SD}$ & 1.97 & 3.73 & 0.91 & 0.13 & 0.17 & 0.07 & 2.05 & 3.56 & 1.40 \\
\hline $\mathrm{db}$ rate below $50 \%$ & Mean & 0.99 & 1.43 & 0.62 & 0.09 & 0.10 & 0.06 & 8.49 & 9.59 & 8.28 \\
\hline & $\mathrm{SD}$ & 1.27 & 2.49 & 0.85 & 0.10 & 0.14 & 0.07 & 2.24 & 3.71 & 2.01 \\
\hline $\mathrm{db}$ rate above $50 \%$ & Mean & 2.12 & 2.58 & 0.69 & 0.17 & 0.16 & 0.07 & 10.1 & 11.4 & 8.62 \\
\hline & $\mathrm{SD}$ & 2.12 & 3.62 & 0.80 & 0.14 & 0.18 & 0.07 & 2.63 & 4.18 & 1.87 \\
\hline$\lambda / \Delta$ & & $102 \%$ & $102 \%$ & $102 \%$ & $102 \%$ & $102 \%$ & $102 \%$ & $102 \%$ & $102 \%$ & $102 \%$ \\
\hline pop below $50 \%$ & Mean & 4.46 & 1.88 & 3.72 & 0.28 & 0.13 & 0.24 & 13.5 & 12.3 & 13.5 \\
\hline & $\mathrm{SD}$ & 4.31 & 1.84 & 2.81 & 0.19 & 0.10 & 0.14 & 3.79 & 2.79 & 2.96 \\
\hline pop above $50 \%$ & Mean & 10.97 & 6.45 & 11.08 & 0.53 & 0.34 & 0.53 & 18.9 & 17.3 & 19.7 \\
\hline & $\mathrm{SD}$ & 6.82 & 4.41 & 5.28 & 0.18 & 0.14 & 0.15 & 5.04 & 3.47 & 3.90 \\
\hline $\mathrm{db}$ rate below $50 \%$ & Mean & 5.20 & 3.79 & 7.44 & 0.32 & 0.22 & 0.38 & 14.2 & 14.2 & 16.6 \\
\hline & $\mathrm{SD}$ & 4.30 & 4.06 & 5.99 & 0.19 & 0.17 & 0.22 & 3.75 & 4.23 & 4.94 \\
\hline $\mathrm{db}$ rate above $50 \%$ & Mean & 10.13 & 4.49 & 7.29 & 0.49 & 0.26 & 0.39 & 18.1 & 15.3 & 16.6 \\
\hline & $\mathrm{SD}$ & 7.41 & 4.05 & 5.18 & 0.22 & 0.15 & 0.19 & 5.69 & 3.75 & 4.31 \\
\hline$\lambda / \Delta$ & & $100 \%$ & $98 \%$ & $100 \%$ & $100 \%$ & $98 \%$ & $100 \%$ & $100 \%$ & $98 \%$ & $100 \%$ \\
\hline pop below $50 \%$ & Mean & 3.54 & 0.74 & 2.44 & 0.24 & 0.06 & 0.18 & 12.3 & 9.97 & 12.0 \\
\hline & $\mathrm{SD}$ & 3.80 & 0.84 & 2.05 & 0.18 & 0.06 & 0.12 & 3.56 & 2.20 & 2.58 \\
\hline pop above $50 \%$ & Mean & 10.30 & 2.95 & 8.43 & 0.52 & 0.20 & 0.45 & 18.2 & 13.7 & 17.5 \\
\hline & $\mathrm{SD}$ & 6.41 & 1.77 & 4.35 & 0.19 & 0.09 & 0.15 & 4.74 & 1.84 & 3.30 \\
\hline $\mathrm{db}$ rate below $50 \%$ & Mean & 4.67 & 1.65 & 5.42 & 0.29 & 0.12 & 0.31 & 13.4 & 11.4 & 14.7 \\
\hline & $\mathrm{SD}$ & 4.30 & 1.83 & 4.85 & 0.20 & 0.11 & 0.21 & 3.87 & 2.98 & 4.32 \\
\hline $\mathrm{db}$ rate above $50 \%$ & Mean & 9.07 & 2.02 & 5.39 & 0.46 & 0.15 & 0.32 & 17.0 & 12.3 & 14.8 \\
\hline & $\mathrm{SD}$ & 7.04 & 1.69 & 4.19 & 0.23 & 0.10 & 0.18 & 5.49 & 2.43 & 3.76 \\
\hline
\end{tabular}

Rows with "pop below 50\%" and "pop above 50\%" report estimates using samples with markets whose population is below or above the median, respectively. Rows with "db rates below $50 \%$ " and "db rate above $50 \%$ " report estimates using samples with markets whose diabetes prevalence rates are above or below the median.

Our estimates suggest that the strategic interaction between providers leads to a downward-sloping reaction curve in capacity choices. A dialysis provider's choice of capacity decreases with that of competitors. A 1 unit increase in the expected capacity of a competitor reduces a provider's entry probability rather substantially. This suggests that conventional models of binary entry decisions would overlook the heterogeneity of strategic effects on providers' capacity choices and market presence. Our counterfactual analyses suggest that providers' responses to payment policies are heterogeneous and the strategic interaction between the choices of capacity plays a significant part in determining 
these responses. Our econometric method is also of interest in its own right and can be applied to a wider class of Bayesian games with continuous choices.

\section{REFERENCES}

Aradillas-Lopez, Andres, "Semiparametric Estimation of a Simultaneous Game with Incomplete Information," Journal of Econometrics 157 (2010), 409-431.

Athey, Susan, "Single Crossing Properties and the Existence of Pure Strategy Equilibria in Games of Incomplete Information," Econometrica 69:4 (2001), 861-890.

Bajari, Patrick, Han Hong, John Krainer, and Denis Nekipelov, "Estimating Static Models of Strategic Interactions," Journal of Business and Economic Statistics 28:4 (2010), 469-482.

Berry, Steven, and Elie Tamer, "Identification in Models of Oligopoly Entry," in Richard Blundell, Whitney K. Newey, and Torsten Persson, eds., Advances in Economics and Econometrics: Theory and Applications (Ninth World Congress), vol. 2 (Cambridge: Cambridge University Press, 2006).

Bresnahan, Timothy, and Peter Reiss, "Entry and Competition in Concentrated Markets," Journal of Political Economy 99 (1991), 977-1009.

Buckley, Jonathan, and Ian James, "Linear Regression with Censored Data," Biometrika 66:3 (1979), 429-436.

Cameron, A. Colin, and Pravin K. Trivedi, Regression Analysis of Count Data (Cambridge: Cambridge University Press, 1998).

Christensen, Ronald, Log-Linear Models and Logistic Regression, 2nd ed. (New York: Springer-Verlag, 1997).

Ciliberto, Frederico, and Ellie Tamer, "Market Structure and Multiple Equilibria in Airline Markets," Econometrica 77 (2009), 1791-1828.

Collard-Wexler, Allan, "Demand Fluctuations in the Ready-Mix Concrete Industry," Econometrica 81 (2013), 1003-1037

Cutler, David, Leemore Dafny, and Christopher Ody, "How Does Competition Impact the Quality of Health Care? A Case Study of the U.S. Dialysis Industry," unpublished manuscript (2012).

Dartmouth Medical School, Center for Evaluative Clinical Sciences, The Dartmouth Atlas of Health Care (Chicago: American Hospital Publishing, 2006).

Davis, Peter, "Spatial Competition in Retail Markets: Movie Theaters," RAND Journal of Economics 37:4 (2006), 964-982.

Dixit, Avinash, "The Role of Investment in Entry-Deterrence," Economic Journal 90 (1980), 95-106.

Ford, Jon, and David Kaserman, "Certificate-of-Need Regulation and Entry: Evidence from the Dialysis Industry," Southern Economic Journal 59:4 (1993), 783-791.

Gelman, Judith, and Steven Salop, "Judo Economics: Capacity Limitation and Coupon Competition," Bell Journal of Economics 14:2 (1983), $315-325$.

Grieco, Paul, and Ryan McDevitt, "Productivity and Quality in Health Care: Evidence from the Dialysis Industry," unpublished manuscript (2014).

Horowitz, Joel, “A Distribution-Free Least Squares Estimator for Censored Linear Regression Models," Journal of Econometrics 32:1 (1986), 59-84.

Jia, Panle, "What Happens When Wal-Mart Comes to Town: An Empirical Analysis of the Discount Retailing Industry," Econometrica 76 (2008), 1263-1316.

Johansen, Kirsten, "Choice of Dialysis Modality in the United States," Archives of Internal Medicine 171:2 (2011), 107-109.

Judd, Kenneth, and Che-Lin Su, "Constrained Optimization Approaches to Estimation of Structural Models," Econometrica 80:5 (2012), 2213 2230.

Kreps, David, and Jose Scheinkman, "Quantity Precommitment and Bertrand Competition Yield Cournot Outcomes," Bell Journal of Economics 14:2 (1983), 326-337.

Mazzeo, Michael, "Product Choice and Oligopoly Market Structure," Rand Journal of Economics 33:2 (2002), 221-242.

Newey, Whitney, and Daniel McFadden, "Large Sample Estimation and Hypothesis Testing," in Robert Engle and Daniel McFadden, eds., Handbook of Econometrics, vol. 4 (Oxford: Elsevier, 1994).

Pollack, Andrew, “The Dialysis Business: Fair Treatment," New York Times, September 16, 2007.
Powell, James, "Least Absolute Deviations Estimation for the Censored Regression Model," Journal of Econometrics 25 (1984), 303-325. "Symmetrically Trimmed Least Squares Estimation of Tobit Models," Econometrica 54 (1986), 1435-1460.

Ramanarayanan, Subramaniam, and Jason Snyder, "Reputations and Firm Performance: Evidence from the Dialysis Industry," unpublished manuscript (2011).

Rust, John, "Optimal Replacement of GMC Bus Engines: An Empirical Model of Harold Zurcher," Econometrica 55:5 (1987), 999-1033.

Schaumans, Catherine, and Frank Verboven, "Entry and Regulation: Evidence from Health Care Professions," RAND Journal of Economics 39:4 (2008), 949-972.

Seim, Katja, "An Empirical Model of Firm Entry with Endogenous Product-Type Choices," RAND Journal of Economics 37:3 (2006), 619-640.

Sweeting, Andrew, "The Strategic Timing Incentives of Commercial Radio Stations: An Empirical Analysis Using Multiple Equilibria," RAND Journal of Economics 40:4 (2009), 710-742.

Thamer, Mae, Yi Zhang, James Kaufman, Dennis Cotter, Fan Dong, and Miguel Hernán, "Dialysis Facility Ownership and Epoetin Dosing in Patients Receiving Hemodialysis," Journal of the American Medical Association 297:15 (2007), 1667-1674.

U.S. Renal Data System, USRDS 2009 Annual Data Report: Atlas of Chronic Kidney Disease and End-Stage Renal Disease in the United States (Bethesda, MD: National Institutes of Health, National Institute of Diabetes and Digestive and Kidney Diseases, 2009).

\section{APPENDIX A}

\section{Supplemental Tables}

TABLE A1.-ADDITIONAL RoBUSTNESS

\begin{tabular}{|c|c|c|c|c|c|c|}
\hline & \multicolumn{2}{|c|}{ FMC } & \multicolumn{2}{|c|}{ DaVita } & \multicolumn{2}{|c|}{ Nonchain } \\
\hline & EST & SE & EST & SE & EST & $\mathrm{SE}$ \\
\hline & \multicolumn{6}{|c|}{ Panel $\mathrm{A}^{\mathrm{a}}$} \\
\hline $\mathrm{K}_{f m c}$ & & & -0.82 & $0.17^{* * *}$ & & \\
\hline $\mathrm{K}_{d a v}$ & -1.14 & $0.20^{* * *}$ & & & -0.88 & $0.17^{* * *}$ \\
\hline $\mathrm{K}_{\text {nonchain }}$ & -1.18 & $0.29^{* * *}$ & -0.60 & $0.28^{* *}$ & -0.55 & $0.18^{* * *}$ \\
\hline lpop & 25.1 & $1.64^{* * *}$ & 19.7 & $1.77^{* * *}$ & 20.7 & $1.48^{* * *}$ \\
\hline black & 51.5 & $15.1^{* * *}$ & 9.54 & 12.4 & 0.76 & 11.7 \\
\hline white & 10.1 & 15.2 & -17.6 & 11.8 & -23.3 & $11.4^{* *}$ \\
\hline latino & 26.5 & $6.98^{* * *}$ & 3.23 & 7.85 & 14.8 & $6.92^{* *}$ \\
\hline asian & -85.2 & $33.1^{* * *}$ & 7.93 & 29.2 & -0.75 & 27.0 \\
\hline age 1 & 28.1 & 36.8 & -60.9 & 38.2 & -39.5 & 36.1 \\
\hline age2 & 38.1 & 33.3 & -54.1 & 34.2 & -20.4 & 31.9 \\
\hline age3 & 36.0 & 32.8 & -20.4 & 34.7 & 24.7 & 32.1 \\
\hline neph & 2.23 & $0.55^{* * *}$ & 2.54 & $0.59^{* * *}$ & 1.32 & $0.59^{* *}$ \\
\hline lbed & 5.53 & $3.08^{*}$ & -2.14 & 3.32 & 6.12 & $3.11^{* *}$ \\
\hline $\mathrm{rn}$ & 10.4 & $3.47^{* * *}$ & 6.35 & $3.69^{*}$ & 3.59 & 3.47 \\
\hline dbrate & 1.67 & $0.64^{* * *}$ & 1.13 & 0.69 & 0.59 & 0.66 \\
\hline conreg & -3.42 & $1.56^{* *}$ & -2.37 & 1.70 & -3.68 & $1.59^{* *}$ \\
\hline $\mathrm{NE}$ & -7.63 & $3.87^{* *}$ & -12.4 & $4.07^{* * *}$ & 7.76 & $3.64 * *$ \\
\hline MW & -6.10 & $2.18^{* * *}$ & -3.09 & 2.36 & -1.94 & 2.26 \\
\hline West & -10.0 & $3.64^{* * *}$ & -15.0 & $4.10^{* * *}$ & 11.8 & $3.11^{* * *}$ \\
\hline dfmc & 13.1 & 8.44 & 14.9 & $8.79^{*}$ & 33.1 & $8.17^{* * *}$ \\
\hline dfmc2 & -3.53 & 2.82 & -2.16 & 2.94 & -12.5 & $2.57^{* * *}$ \\
\hline ddav & 2.38 & 11.5 & -21.5 & $11.6^{*}$ & 58.7 & $11.0^{* * *}$ \\
\hline ddav2 & 0.35 & 5.69 & 15.4 & $5.72^{* * *}$ & -26.0 & $5.45^{\text {*** }}$ \\
\hline cons & -358 & $35.8^{* * *}$ & -192 & $35.9^{* * *}$ & -260 & $31.0^{* * *}$ \\
\hline sigma & 23.0 & $0.71^{* * *}$ & 23.5 & $0.83^{* * *}$ & 23.0 & $0.75^{* * *}$ \\
\hline $\begin{array}{l}\text { Number of } \\
\text { observations }\end{array}$ & 3,129 & & & & & \\
\hline
\end{tabular}




\begin{tabular}{|c|c|c|c|c|c|c|}
\hline & \multicolumn{2}{|c|}{ FMC } & \multicolumn{2}{|c|}{ DaVita } & \multicolumn{2}{|c|}{ Nonchain } \\
\hline & EST & SE & EST & SE & EST & SE \\
\hline & \multicolumn{6}{|c|}{ Panel $\mathrm{B}^{\mathrm{b}}$} \\
\hline $\mathrm{K}_{f m c}$ & & & -0.69 & $0.17^{* * *}$ & & \\
\hline $\mathrm{K}_{d a v}$ & -0.84 & $0.16^{* * *}$ & & & -0.64 & $0.16^{* * *}$ \\
\hline $\mathrm{K}_{\text {nonchain }}$ & -0.92 & $0.25^{* * *}$ & -0.22 & 0.25 & -0.35 & $0.15^{* *}$ \\
\hline lpop & 25.1 & $1.58^{* * *}$ & 19.6 & $1.83^{* * *}$ & 20.5 & $1.48^{* * *}$ \\
\hline black & 58.7 & $16.2^{* * *}$ & 10.0 & 13.0 & -1.53 & 12.1 \\
\hline white & 15.0 & 16.2 & -20.3 & 12.6 & -25.6 & $12.0^{* *}$ \\
\hline latino & 26.3 & $7.02^{* * *}$ & -1.42 & 8.20 & 14.6 & $7.08^{* *}$ \\
\hline asian & -72.2 & $32.3^{* *}$ & 16.6 & 29.7 & -2.27 & 27.6 \\
\hline age1 & 33.0 & 37.5 & -65.2 & 40.1 & -39.3 & 37.2 \\
\hline age 2 & 36.1 & 34.2 & -65.5 & $36.0^{*}$ & -24.6 & 33.0 \\
\hline age 3 & 46.9 & 33.4 & -20.4 & 36.3 & 27.7 & 33.0 \\
\hline neph & 1.98 & $0.57^{* * *}$ & 2.50 & $0.63^{* * *}$ & 1.14 & $0.61^{*}$ \\
\hline lbed & 6.86 & $3.16^{* *}$ & -2.76 & 3.50 & 7.04 & $3.20^{* *}$ \\
\hline $\mathrm{rn}$ & 10.3 & $3.54^{* * *}$ & 6.62 & $3.87^{*}$ & 3.02 & 3.58 \\
\hline dbrate & 1.47 & $0.65^{* *}$ & 0.89 & 0.72 & 0.27 & 0.68 \\
\hline conreg & -3.76 & $1.60^{* *}$ & -2.59 & 1.78 & -3.88 & $1.63^{* *}$ \\
\hline $\mathrm{NE}$ & -10.2 & $3.95^{* * *}$ & -17.3 & $4.22^{* * *}$ & 9.05 & $3.72^{* *}$ \\
\hline MW & -6.23 & $2.19^{* * *}$ & -3.46 & 2.43 & -1.18 & 2.29 \\
\hline West & -10.8 & $3.65^{* * *}$ & -16.6 & $4.29^{* * *}$ & 13.2 & $3.20^{* * *}$ \\
\hline dfmc & 7.29 & 8.36 & 10.3 & 9.02 & 34.2 & $8.18^{* * *}$ \\
\hline $\mathrm{dfmc} 2$ & -1.83 & 2.80 & -0.64 & 3.04 & -13.1 & $2.59^{* * *}$ \\
\hline ddav & -1.29 & 11.4 & -29.0 & $12.0^{* *}$ & 61.9 & $11.2^{* * *}$ \\
\hline ddav2 & 1.22 & 5.64 & 19.4 & $5.87^{* * *}$ & -27.3 & $5.52^{* * *}$ \\
\hline cons & -360 & $36.3^{* * *}$ & -177 & $37.1^{* * *}$ & -257 & $31.4^{* * *}$ \\
\hline sigma & 23.9 & $0.71^{* * *}$ & 25.0 & $0.83^{* * *}$ & 24.1 & $0.76^{* * *}$ \\
\hline \multicolumn{6}{|l|}{ Number of } & \\
\hline \multicolumn{7}{|c|}{ Panel $C^{c}$} \\
\hline $\mathrm{K}_{f m c}$ & & & -0.75 & $0.23^{* * *}$ & & \\
\hline $\mathrm{K}_{d a v}$ & -1.46 & $0.28^{* * *}$ & & & -1.05 & $0.23^{* * *}$ \\
\hline $\mathrm{K}_{\text {nonchain }}$ & -1.45 & $0.37^{* * *}$ & -0.93 & $0.37^{* *}$ & -0.93 & $0.28^{* * *}$ \\
\hline lpop & 21.6 & $1.61^{* * *}$ & 17.0 & $1.79^{* * *}$ & 18.6 & $1.45^{* * *}$ \\
\hline black & 42.4 & $13.0^{* * *}$ & 8.45 & 11.9 & 4.78 & 10.9 \\
\hline white & 4.95 & 13.1 & -13.4 & 11.2 & -20.7 & $10.6^{*}$ \\
\hline latino & 19.4 & $6.59^{* * *}$ & -1.22 & 8.04 & 5.67 & 7.13 \\
\hline asian & -77.3 & $31.6^{* *}$ & 32.4 & 29.2 & -1.86 & 26.8 \\
\hline age 1 & 22.4 & 34.3 & -78.1 & $38.1^{\text {** }}$ & -47.4 & 35.9 \\
\hline age2 & 21.6 & 30.7 & -69.7 & $33.1^{\text {** }}$ & -26.1 & 30.7 \\
\hline age 3 & 32.1 & 30.6 & -26.4 & 34.4 & 12.9 & 31.6 \\
\hline neph & 2.26 & $0.50^{* * *}$ & 2.16 & $0.59^{* * *}$ & 1.41 & $0.57^{* *}$ \\
\hline lbed & 4.17 & 2.86 & -4.25 & 3.30 & 4.41 & 2.99 \\
\hline $\mathrm{rn}$ & 8.68 & $3.23^{* * *}$ & 6.70 & $3.61^{*}$ & 3.26 & 3.35 \\
\hline dbrate & 1.52 & $0.59^{* * *}$ & 1.15 & $0.69^{*}$ & 0.75 & 0.65 \\
\hline conreg & -2.79 & $1.46^{*}$ & -1.60 & 1.69 & -3.70 & $1.54^{* *}$ \\
\hline $\mathrm{NE}$ & -9.05 & $3.67^{* *}$ & -11.3 & $4.15^{* * *}$ & 3.77 & 3.73 \\
\hline MW & -6.49 & $2.03^{* * *}$ & -4.47 & $2.34^{*}$ & -1.43 & 2.23 \\
\hline West & -6.85 & $3.57^{*}$ & -11.1 & $4.14^{* * *}$ & 11.5 & $3.03^{* * *}$ \\
\hline dfmc & 8.99 & 7.98 & 16.8 & $8.85^{*}$ & 24.6 & $8.40^{* * *}$ \\
\hline dfmc2 & -2.80 & 2.64 & -3.83 & 2.94 & -9.58 & $2.60^{* * *}$ \\
\hline ddav & -3.53 & 10.4 & -19.5 & $11.2^{*}$ & 43.8 & $10.5^{* * *}$ \\
\hline
\end{tabular}

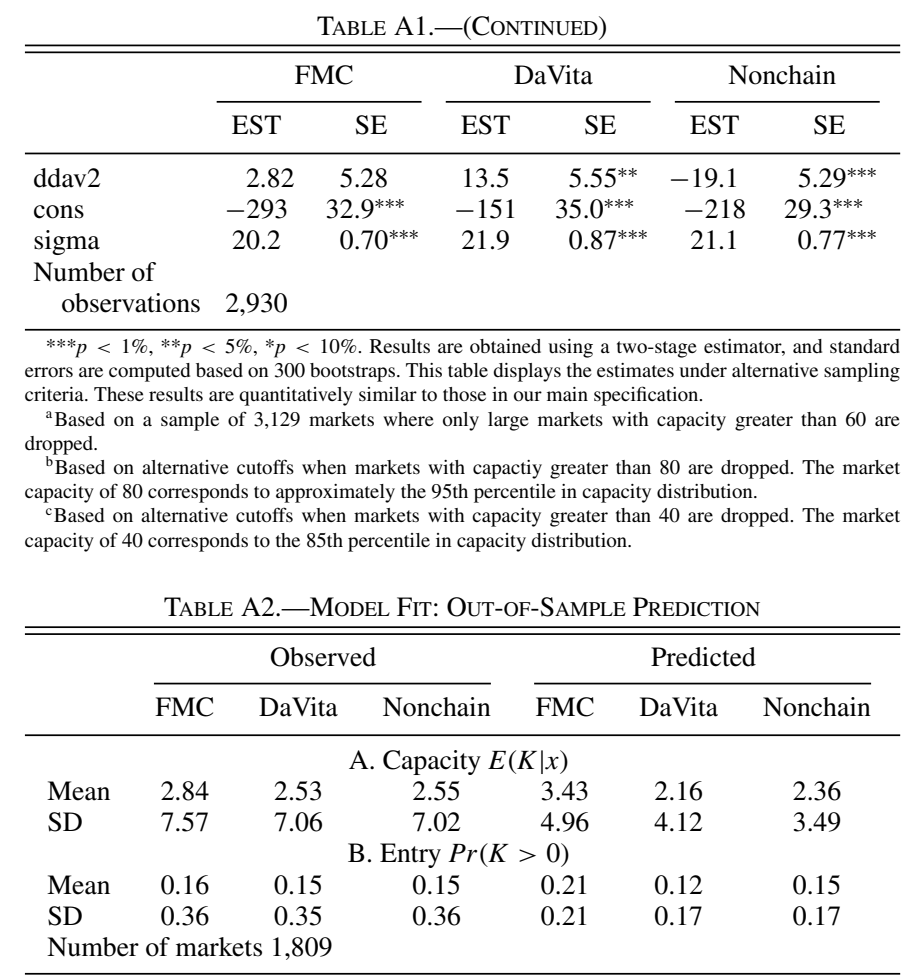

The prediction is made based on the GMM estimates reported in table 4 and the larger sample used for panel A of table A1. We report the results after excluding 1,320 markets that are currently included in the main specifications. This left 3,129-1,320 $=1,809$ markets.

\section{APPENDIX B}

\section{Proof of Proposition 1}

First, suppose that the solution of the maximization problem is in the interior: $K_{i}^{*}\left(X, \varepsilon_{i}\right)>0$. Then the first-order condition of equation (2) implies that in equilibrium,

$$
K_{i}^{*}\left(X, \varepsilon_{i}\right)=\frac{1}{2 a_{i}}\left\{X \beta_{i}+\sum_{j \neq i} \gamma_{i, j} \mathbb{E}_{\varepsilon_{j}}\left[K_{j}^{*}\left(X, \varepsilon_{j}\right) \mid x, \varepsilon_{i}\right]-b_{i}-\varepsilon_{i}\right\},
$$

where the right-hand side of equation (A1) is necessarily strictly positive. Since $a_{i}>0$, the second-order condition for such an interior solution would be satisfied automatically. Next, suppose the solution is on the corner: $K_{i}^{*}\left(X, \varepsilon_{i}\right)=0$. Because $a_{i}>0$ and $\Pi_{i}\left(0, K_{-i}, x, \varepsilon_{i}\right)=0$ regardless of $K_{-i}$, the first-order condition of equation (2) evaluated at $K_{i}^{*}\left(X, \varepsilon_{i}\right)=0$ and the competitors' strategies $K_{-i}^{*}($.) is necessarily negative. (Otherwise the solution would be interior.) Therefore, with a corner solution in equilibrium, we have

$$
K_{i}^{*}\left(X, \varepsilon_{i}\right)=0 \text { and } X \beta_{i}+\sum_{j \neq i} \gamma_{i, j} \mathbb{E}_{\varepsilon_{j}}\left[K_{j}^{*}\left(X, \varepsilon_{j}\right) \mid x, \varepsilon_{i}\right]-b_{i}-\varepsilon_{i}<0 .
$$

Combining equations (A1) and (A2), we conclude that equation (3) holds in any PSBNE. 